\title{
Phase Diffusion in Quantum Dissipative Systems
}

\author{
Subhashish Banerjee* \\ Raman Research Institute, Sadashiva Nagar, Bangalore - 560 080, India \\ R. Srikanth† \\ Poornaprajna Institute of Scientific Research, Devanahalli, Bangalore- 562 110, India and \\ Raman Research Institute, Sadashiva Nagar, Bangalore - 560 080, India
}

\begin{abstract}
We study the dynamics of the quantum phase distribution associated with the reduced density matrix of a system for a number of situations of practical importance, as the system evolves under the influence of its environment, interacting via a quantum nondemoliton type of coupling, such that there is decoherence without dissipation, as well as when it interacts via a dissipative interaction, resulting in decoherence as well as dissipation. The system is taken to be either a two-level atom (or equivalently, a spin-1/2 system) or a harmonic oscillator, and the environment is modeled as a bath of harmonic oscillators, starting out in a squeezed thermal state. The impact of the different environmental parameters on the dynamics of the quantum phase distribution for the system starting out in various initial states, is explicitly brought out. An interesting feature that emerges from our work is that the relationship between squeezing and temperature effects depends on the type of system-bath interaction. In the case of quantum nondemolition type of interaction, squeezing and temperature work in tandem, producing a diffusive effect on the phase distribution. In contrast, in case of a dissipative interaction, the influence of temperature can be counteracted by squeezing, which manifests as a resistence to randomization of phase. We make use of the phase distributions to bring out a notion of complementarity in atomic systems. We also study the dispersion of the phase using the phase distributions conditioned on particular initial states of the system.
\end{abstract}

PACS numbers: 03.65.Yz, 42.50.Ct

\section{INTRODUCTION}

Open quantum systems are ubiquitous in the sense that any system can be thought of as being surrounded by its environment (reservoir or bath) which influences its dynamics. They provide a natural route for discussing damping and dephasing. One of the first testing grounds for open system ideas was in quantum optics [1]. Its application to other areas gained momentum from the works of Caldeira and Leggett [2], and Zurek [3], among others. Depending upon the system-reservoir $(S-R)$ interaction, open systems can be broadly classified into two categories, viz., quantum non-demolition (QND) or dissipative. A particular type of quantum nondemolition (QND) $S-R$ interaction is given by a class of energy-preserving measurements in which dephasing occurs without damping the system. This may be achieved when the Hamiltonian $H_{S}$ of the system commutes with the Hamiltonian $H_{S R}$ describing the systemreservoir interaction, i.e., $H_{S R}$ is a constant of the motion generated by $H_{S}[4,[5,6]$. A dissipative open system would be when $H_{S}$ and $H_{S R}$ do not commute resulting in dephasing along with damping [7]. A prototype of dissipative open quantum systems, having many applications, is the quantum Brownian motion of harmonic oscillators. This model was studied by Caldeira and Leggett [2] for the case where the system and its environment were initially separable. The above treatment of the quantum Brownian motion was generalized to the physically reasonable initial condition of a mixed state of the system and its environment by Hakim and Ambegaokar 8], Smith and Caldeira [9], Grabert, Schramm and Ingold [10], and for the case of a system in a Stern-Gerlach potential [1]], and also for the quantum Brownian motion with nonlinear system-environment couplings [12], among others.

The interest in the relevance of open system ideas to quantum information has increased in recent times because of the impressive progress made on the experimental front in the manipulation of quantum states of matter towards quantum information processing and quantum communication. Myatt et al. [13] and Turchette et al. [14] have performed a series of experiments in which they induced decoherence and decay by coupling the atom (their system- $S$ ) to engineered reservoirs, in which the coupling to, and the state of, the environment are controllable. An experiment reported in Ref. [15] demonstrated and completely characterized a QND scheme for making a nondeterministic

\footnotetext{
*Electronic address: subhashishb@rri.res.in
}

${ }^{\dagger}$ Electronic address: srik@rri.res.in 
measurement of a single photon nondestructively using only linear optics and photo-detection of ancillary modes, to induce a strong nonlinearity at the single photon level. The dynamics of decoherence in continuous atom-optical QND measurements has been studied by Onofrio and Viola [16]. In addition to its relevance in ultrasensitive measurements, a QND scheme provides a way to prepare quantum mechanical states which may otherwise be difficult to create, such as Fock states with a specific number of particles. It has been shown that the accuracy of atomic interferometry can be improved by using QND measurements of the atomic populations at the inputs to the interferometer [17]. QND systems have also been proposed for engineering quantum dynamical evolution of a system with the help of a quantum meter [18]. In a recent study of QND open system Hamiltonians for two different models of the environment describable as baths of either oscillators or spins, an interesting connection was found between the energy-preserving QND Hamiltonians and the phase space area-preserving canonical transformations [19].

A class of observables that may be measured repeatedly with arbitrary precision, with the influence of the measurement apparatus on the system being confined strictly to the conjugate observables, is called QND or back-action evasive observables [20, 21, 22, 23]. Such a measurement scheme was originally introduced in the context of the detection of gravitational waves [24, 25]. The energy preserving measurements, referred to above, form an important class of such a general QND measurement scheme. Since they describe dephasing without dissipation, a study of phase diffusion in such a situation is important from the context of a number of experimental situations.

The quantum description of phases [26, 27] has a long history [28, 29, 30, 31, 32]. Pegg and Barnett [31], following Dirac [28], carried out a polar decomposition of the annihilation operator and defined a hermitian phase operator in a finite-dimensional Hilbert space. In their scheme, the expectation value of a function of the phase operator is first carried out in a finite-dimensional Hilbert space, and then the dimension is taken to the limit of infinity. However, it is not possible to interpret this expectation value as that of a function of a hermitian phase operator in an infinitedimensional Hilbert space [33, 34]. To circumvent this problem, the concept of phase distribution for the quantum phase has been introduced [33, 35]. In this scheme, one associates a phase distribution to a given state such that the average of a function of the phase operator in the state, computed with the phase distribution, reproduces the results of Pegg and Barnett.

A study of the quantum phase diffusion in a number of QND systems was carried out in Ref. [36] using the phase distribution approach. In this work we extend the above study to include the effect of dissipation on phase diffusion. Throughout this paper, the bath is assumed to be a collection of harmonic oscillators starting from a squeezed thermal initial state. An advantage of using a squeezed thermal bath is that the decay rate of quantum coherences can be suppressed leading to preservation of non-classical effects [37, 38, 39]. It has also been shown to modify the evolution of the geometric phase of two-level atomic systems [40]. The plan of the paper is as follows. In Section II, we recollect some results on the quantum phase distribution in QND systems from [36, 39]. We extend the previous expressions, for a single two-level atomic system, to the case of two two-level atoms and further plot the quantum phase distribution for ten two-level atoms. Following Agarwal and Singh [41] we also introduce the number distribution and use it to discuss the complementary between the number and phase distributions. In Section III, we study the quantum phase distribution of a two-level atomic system interacting with its bath via a dissipative interaction. The evolution is governed by a Lindblad equation. The phase distribution is studied for the system initially (a) in an atomic coherent state and (b) in an atomic squeezed state. For the system in an atomic coherent state, complementarity between the number and phase distributions is discussed. In Section IV, the quantum phase distribution of the system of a harmonic oscillator, in a dissipative interaction with its bath, is obtained. In Section V, an application is made of the quantum phase distributions obtained for various initial system states and $S-R$ interactions, to study the corresponding phase dispersion. In Section VI, we present our conclusions.

\section{QUANTUM PHASE DISTRIBUTION: QND}

Here we recapulate, from [36], the results of Quantum Phase Distributions for a two-level atomic system as well as that of a harmonic oscillator which undergo interaction with their environments via a QND type of interaction. We consider the following Hamiltonian which models the interaction of a system with its environment, modeled as a bath of harmonic oscillators, via a QND type of coupling [39]:

$$
\begin{aligned}
H & =H_{S}+H_{R}+H_{S R} \\
& =H_{S}+\sum_{k} \hbar \omega_{k} b_{k}^{\dagger} b_{k}+H_{S} \sum_{k} g_{k}\left(b_{k}+b_{k}^{\dagger}\right)+H_{S}^{2} \sum_{k} \frac{g_{k}^{2}}{\hbar \omega_{k}} .
\end{aligned}
$$

Here $H_{S}, H_{R}$ and $H_{S R}$ stand for the Hamiltonians of the system, reservoir and system-reservoir interaction, respectively. The $g_{k}$ 's are dimensionless coupling constants. The last term on the right-hand side of Eq. (1) is a renormalization inducing 'counter term'. Since $\left[H_{S}, H_{S R}\right]=0,(1)$ is of QND type. Here $H_{S}$ is a generic system 
Hamiltonian which will be used subsequently to model different physical situations. Assuming separable initial conditions with the bath being initially in a squeezed thermal state and tracing over the bath degrees of freedom, the reduced density matrix of the system of interest $S$, in the system eigenbasis, is obtained as [39]

$$
\rho_{n m}^{s}(t)=e^{-\frac{i}{\hbar}\left(E_{n}-E_{m}\right) t} e^{i\left(E_{n}^{2}-E_{m}^{2}\right) \eta(t)} \times \exp \left[-\left(E_{m}-E_{n}\right)^{2} \gamma(t)\right] \rho_{n m}^{s}(0)
$$

where

$$
\eta(t)=-\sum_{k} \frac{g_{k}^{2}}{\hbar^{2} \omega_{k}^{2}} \sin \left(\omega_{k} t\right)
$$

and

$$
\gamma(t)=\frac{1}{2} \sum_{k} \frac{g_{k}^{2}}{\hbar^{2} \omega_{k}^{2}} \operatorname{coth}\left(\frac{\beta \hbar \omega_{k}}{2}\right)\left|\left(e^{i \omega_{k} t}-1\right) \cosh \left(r_{k}\right)+\left(e^{-i \omega_{k} t}-1\right) \sinh \left(r_{k}\right) e^{i 2 \Phi_{k}}\right|^{2} .
$$

For the reservoir $R$ to be considered as a proper bath causing decoherence and (possibly) dissipation, we need to assume a 'quasi-continuous' bath spectrum with spectral density $I(\omega)$ such that for an arbitrary function $f(\omega)$ the continuum limit implies [6]

$$
\sum_{k} \frac{g_{k}^{2}}{\hbar^{2}} f\left(\omega_{k}\right) \longrightarrow \int_{0}^{\infty} d \omega I(\omega) f(\omega)
$$

We consider the case of an Ohmic bath with spectral density

$$
I(\omega)=\frac{\gamma_{0}}{\pi} \omega e^{-\omega / \omega_{c}}
$$

where $\gamma_{0}$, having the dimension of $1 /(\text { energy })^{2}[6]$, and $\omega_{c}$ are two bath parameters characterizing the quantum noise. Using Eqs. (5) and (6) in Eq. (3), we obtain [39]

$$
\eta(t)=-\frac{\gamma_{0}}{\pi} \tan ^{-1}\left(\omega_{c} t\right)
$$

Using Eqs. (5), (6) in Eq. (44) and using the $T=0$ limit, $\gamma(t)$ is obtained as [39]

$$
\begin{aligned}
\gamma(t)= & \frac{\gamma_{0}}{2 \pi} \cosh (2 r) \ln \left(1+\omega_{c}^{2} t^{2}\right)-\frac{\gamma_{0}}{4 \pi} \sinh (2 r) \ln \left[\frac{\left(1+4 \omega_{c}^{2}(t-a)^{2}\right)}{\left(1+\omega_{c}^{2}(t-2 a)^{2}\right)^{2}}\right] \\
& -\frac{\gamma_{0}}{4 \pi} \sinh (2 r) \ln \left(1+4 a^{2} \omega_{c}^{2}\right),
\end{aligned}
$$

where the resulting integrals are defined only for $t>2 a$ [42]. Using Eqs. (5), (6) in Eq. (44) and using the high $T$ limit, $\gamma(t)$ is obtained as 39]

$$
\begin{aligned}
\gamma(t)= & \frac{\gamma_{0} k_{B} T}{\pi \hbar \omega_{c}} \cosh (2 r)\left[2 \omega_{c} t \tan ^{-1}\left(\omega_{c} t\right)+\ln \left(\frac{1}{1+\omega_{c}^{2} t^{2}}\right)\right] \\
& -\frac{\gamma_{0} k_{B} T}{2 \pi \hbar \omega_{c}} \sinh (2 r)\left[4 \omega_{c}(t-a) \tan ^{-1}\left(2 \omega_{c}(t-a)\right)\right. \\
& -4 \omega_{c}(t-2 a) \tan ^{-1}\left(\omega_{c}(t-2 a)\right)+4 a \omega_{c} \tan ^{-1}\left(2 a \omega_{c}\right) \\
& \left.+\ln \left(\frac{\left[1+\omega_{c}^{2}(t-2 a)^{2}\right]^{2}}{\left[1+4 \omega_{c}^{2}(t-a)^{2}\right]}\right)+\ln \left(\frac{1}{1+4 a^{2} \omega_{c}^{2}}\right)\right],
\end{aligned}
$$

where, again, the resulting integrals are defined for $t>2 a$ [42]. Here we have for simplicity taken the squeezed bath parameters as

$$
\begin{aligned}
\cosh (2 r(\omega)) & =\cosh (2 r), \quad \sinh (2 r(\omega))=\sinh (2 r), \\
\Phi(\omega) & =a \omega,
\end{aligned}
$$

where $a$ is a constant depending upon the squeezed bath. Note that the results pertaining to a thermal bath can be obtained from the above equations by setting the squeezing parameters $r$ and $\Phi$ to zero. It is interesting to note that in the context of quantum information, the open system effect depicted in this Section can be modeled by a familiar quantum noisy channel, viz., the phase damping channel [40, 43, 44]. 


\section{A. Two-Level Atomic Systems}

Here we consider the case where our system $S$ is a two-level atom. The system Hamiltonian $H_{S}$ is

$$
H_{S}=\frac{\hbar \omega}{2} \sigma_{z}
$$

where $\sigma_{z}$ is the usual Pauli matrix. The form of the system Hamiltonian $H_{S}$, Eq. (11), when substituted in Eq. (1) has been used in the context of quantum computation [45, 46, 47]. In the context of a system of multiple two-level atoms, which is equivalent to an angular momentum system, we set $H_{S}=\hbar \omega J_{z}$. The Wigner-Dicke state [48, 49, 50] $|j, m\rangle$, which are the simultaneous eigenstates of the angular momentum operators $J^{2}$ and $J_{z}$, serve as the basis states for $H_{S}$, and we have

$$
\begin{aligned}
H_{S}|j, m\rangle & =\hbar \omega m|j, m\rangle \\
& =E_{j, m}|j, m\rangle .
\end{aligned}
$$

Here $-j \leq m \leq j$. Using this basis and the above equation in Eq. (2) we obtain the reduced density matrix of the system as

$$
\rho_{j m, j n}^{s}(t)=e^{-i \omega(m-n) t} e^{i(\hbar \omega)^{2}\left(m^{2}-n^{2}\right) \eta(t)} e^{-(\hbar \omega)^{2}(m-n)^{2} \gamma(t)} \rho_{j m, j n}^{s}(0) .
$$

Following Agarwal and Singh [41] we introduce the phase distribution $\mathcal{P}(\phi), \phi$ being related to the phase of the dipole moment of the system, as

$$
\mathcal{P}(\phi)=\frac{2 j+1}{4 \pi} \int_{0}^{\pi} d \theta \sin (\theta) Q(\theta, \phi)
$$

where $\mathcal{P}(\phi)>0$ and is normalized to unity, i.e., $\int_{0}^{2 \pi} d \phi \mathcal{P}(\phi)=1$. Here $Q(\theta, \phi)$ is defined as

$$
Q(\theta, \phi)=\left\langle\theta, \phi\left|\rho^{s}\right| \theta, \phi\right\rangle,
$$

where $|\theta, \phi\rangle$ are the atomic coherent states [51, 52] given by an expansion over the Wigner-Dicke states [49] as

$$
|\theta, \phi\rangle=\sum_{m=-j}^{j}\left(\begin{array}{c}
2 j \\
j+m
\end{array}\right)^{\frac{1}{2}}(\sin (\theta / 2))^{j+m}(\cos (\theta / 2))^{j-m}|j, m\rangle e^{-i(j+m) \phi}
$$

Using Eq. (15) in Eq. (14), with insertions of partitions of unity in terms of the Wigner-Dicke states, we can write the phase distribution function as

$$
\begin{aligned}
\mathcal{P}(\phi)= & \frac{2 j+1}{4 \pi} \int_{0}^{\pi} d \theta \sin \theta \sum_{n, m=-j}^{j}\langle\theta, \phi \mid j, n\rangle \\
& \times\left\langle j, n\left|\rho^{s}(t)\right| j, m\right\rangle\langle j, m \mid \theta, \phi\rangle .
\end{aligned}
$$

Now we take up two physically interesting initial conditions for the system $S$.

\section{System initially in an atomic coherent state}

Here we consider the system $S$ to be initially in an atomic coherent state which is the atomic analogue of the Glauber coherent state [49]. Thus the initial system density matrix is

$$
\rho^{s}(0)=\left|\alpha^{\prime}, \beta^{\prime}\right\rangle\left\langle\alpha^{\prime}, \beta^{\prime}\right|
$$

Using the Eqs. (13), (18) in Eq. (17) we obtain the phase distribution for a two-level atom, with $j=\frac{1}{2}$ as 36 ]

$$
\mathcal{P}(\phi)=\frac{1}{2 \pi}\left[1+\frac{\pi}{4} \sin \left(\alpha^{\prime}\right) \cos \left(\beta^{\prime}+\omega t-\phi\right) e^{-(\hbar \omega)^{2} \gamma(t)}\right] .
$$

It can be easily checked that this $\mathcal{P}(\phi)$ is normalized to unity. As can be seen from Eq. (19), only $\gamma(t)$ plays a role in the effect of the environment on the phase distribution. 


\section{System initially in an atomic squeezed state}

Now we consider our system $S$ to be initially in an atomic squeezed state [51, 52, 53, 54] expressed in terms of the Wigner-Dicke states as

$$
\left.|\zeta, p\rangle=A_{p} \exp \left(\Theta J_{z}\right) \exp \left(-i \frac{\pi}{2} J_{y}\right)\right)|j, p\rangle
$$

where

$$
e^{2 \Theta}=\tanh (2|\zeta|)
$$

and $A_{p}$ is usually obtained by normalization. Thus the initial density matrix of the system $S$ is

$$
\rho^{s}(0)=|\zeta, p\rangle\langle\zeta, p| .
$$

Using the Eqs. (13), (22) in Eq. (17) we obtain the phase distribution for a two-level atom, with $j=\frac{1}{2}$ for $p= \pm \frac{1}{2}$ as [36]

$$
\mathcal{P}(\phi)=\frac{1}{2 \pi}\left[1 \pm \frac{\pi}{4 \cosh (\Theta)} \cos (\phi-\omega t) e^{-(\hbar \omega)^{2} \gamma(t)}\right]
$$

It can be seen that Eqs. (23), are normalized to unity.

The above expressions may be extended to the case of multiple two-level atoms. For e.g., the quantum phase distribution for two two-level atoms, with $j=1$, is:

$$
\begin{aligned}
\mathcal{P}(\phi) & =\frac{1}{2 \pi}\left\{1 \pm \frac{3 \pi}{4(1+\cosh (2 \Theta))}\left[\cos (\phi-\omega t) \cos \left([\hbar \omega]^{2} \eta(t)\right) \cosh (\Theta)\right.\right. \\
& \left.-\sin (\phi-\omega t) \sin \left([\hbar \omega]^{2} \eta(t)\right) \sinh (\Theta)\right] \exp \left(-[\hbar \omega]^{2} \gamma(t)\right) \\
& \left.+\frac{1}{2(1+\cosh (2 \Theta))} \cos (2[\phi-\omega t]) \exp \left(-4[\hbar \omega]^{2} \gamma(t)\right)\right\}
\end{aligned}
$$

for $p= \pm 1$ and

$$
\mathcal{P}(\phi)=\frac{1}{2 \pi}\left\{1-\frac{1}{2 \cosh (2 \Theta)} \cos (2(\phi-\omega t)) \exp \left(-4[\hbar \omega]^{2} \gamma(t)\right)\right\}
$$

for $p=0$.

In comparison with Eq. (23), which gives the quantum phase distribution for a single two-level atom, it can be seen that Eq. (24) (phase distribution for two two-level atoms) involves both $\eta(t)$ and $\gamma(t)$. This procedure may be carried to any number of two-level atoms using the Wigner- $d$ function [55]:

$$
d_{n, p}^{j}(\pi / 2)=2^{-j} \sqrt{(j+n) !(j-n) !(j+p) !(j-p) !)} \sum_{q} \frac{(-1)^{q}}{q !(j+n-q) !(j-p-q) !(p+q-n) !},
$$

where $d_{n, p}^{j}(\theta)$ is the standard Wigner symbol for the rotation operator [55]

$$
d_{n, p}^{j}(\theta)=\left\langle j, n\left|e^{-i \theta J_{y}}\right| j, p\right\rangle
$$

In Figure 1, we plot the quantum phase distribution for ten two-level atoms. It can be clearly seen from the figure that compared to the unitary case, interaction with the bath (characterized by finiteness of $\gamma_{0}$ ) causes phase diffusion. A comparison of the small- and large-dashed curves indicates that with increase in bath exposure duration $t$, the phase distribution diffuses as well as shifts to the right. It is also evident from the figure that increase in the bath squeezing $r$ and temperature $T$ also cause phase diffusion. The phase distributions are normalized to unity.

In the case of QND type of interaction, the system is decohered without its energy being affected. This is reflected in the fact that with higher noise, the 'phase' gets completely randomized, resulting in a flattening of the distribution $P(\phi)$, as depicted in Figure 1, whereas the 'number' distribution, given by

$$
\begin{aligned}
p(m)= & \left\langle j, m\left|\rho^{s}(t)\right| j, m\right\rangle, \quad|m| \leq j, \\
= & \begin{cases}\left(\begin{array}{c}
2 j \\
j+m
\end{array}\right)\left(\sin \left(\alpha^{\prime} / 2\right)\right)^{2(j+m)}\left(\cos \left(\alpha^{\prime} / 2\right)\right)^{2(j-m)} & \text { for initial atomic coherent state Eq. (18) } \\
\left|A_{p}\right|^{2} e^{2 m \Theta}\left|d_{m p}^{j}(\pi / 2)\right|^{2} & \text { for initial atomic squeezed state Eq. (22), }\end{cases}
\end{aligned}
$$




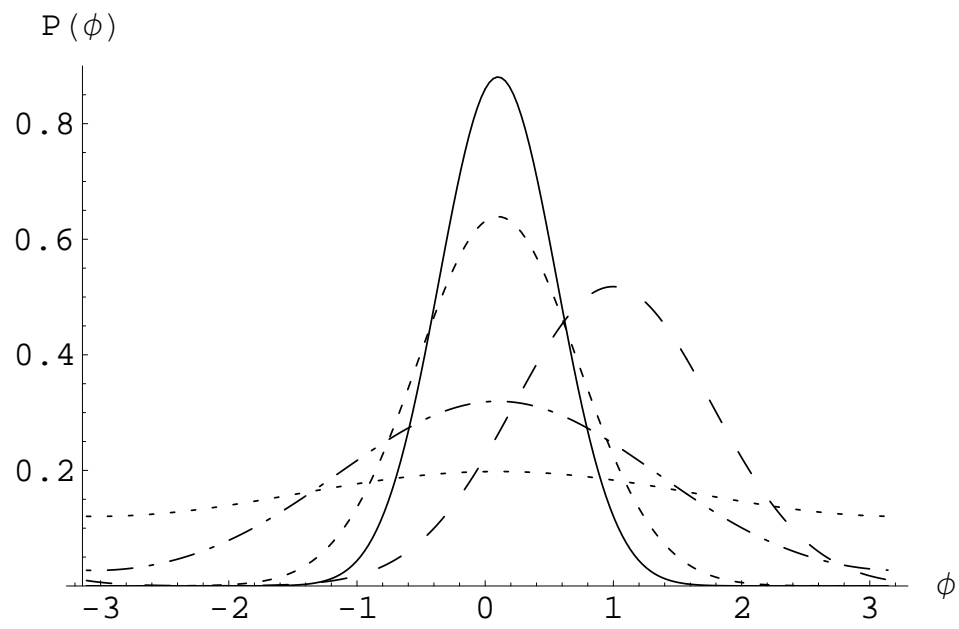

FIG. 1: Quantum phase distribution $\mathcal{P}(\phi)$ with respect to $\phi$ (in radians) for ten atoms, starting in an atomic squeezed state (Eq. (22) ), with $j=p=5, a=0$ (Eq. (10) ), $\Theta=-0.01832$ (Eq. (21)) and $\gamma_{0}=0.025$, undergoing a QND system-bath interaction. Here $\omega=1$ and $\omega_{c}=100$. The bold curve represents unitary evolution for $t=0.1$, while the small-dashed and large-dashed curves are for the bath squeezing parameter $r=1.0$, temperature (in units where $\hbar \equiv k_{B} \equiv 1$ ) $T=0.0$ and evolution times $t=0.1$ and 1 , respectively. The dot-dashed curve represents the case $r=2.0, t=0.1, T=0.0$, and the dotted curve the case $r=1.0, t=0.1, T=300.0$.

remains unaffected. The distributions $p(m)$ and $P(\phi)$ may be thought of as complementary [41] in the sense of conjugate Hermitian observables. For example, it may be verified that a 'number' state, i.e., Wigner-Dicke state, corresponds to a phase distribution of maximum uncertainty (in the entropic sense) [56]. This process may be understood as the selection of states in a preferred pointer basis [3, 5], which in this case are the Wigner-Dicke states, because of the nature of the system-reservoir interaction, whereby the environment 'monitors' the system in the preferred basis. As $p(m)$ represents information in the preferred basis [5], the influence of the environment is not seen explicitly in Eq. (29).

\section{B. Harmonic Oscillator System}

Here the system of interest $S$ is taken to be a harmonic oscillator with the Hamiltonian

$$
H_{S}=\hbar \omega\left(a^{\dagger} a+\frac{1}{2}\right) .
$$

The number states serve as an appropriate basis for the system Hamiltonian and the system energy eigenvalue (30) in this basis is

$$
E_{n}=\hbar \omega\left(n+\frac{1}{2}\right) .
$$

Following Agarwal et al. [35] we define a phase distribution $\mathcal{P}(\theta)$ for a given density operator $\hat{\rho}$ associated with a state $|\theta\rangle$ as

$$
\begin{aligned}
\mathcal{P}(\theta) & =\frac{1}{2 \pi}\langle\theta|\rho| \theta\rangle, 0 \leq \theta \leq 2 \pi, \\
& =\frac{1}{2 \pi} \sum_{m, n=0}^{\infty} \rho_{m, n} e^{i(n-m) \theta},
\end{aligned}
$$

where the states $|\theta\rangle$ are the analogues of the Susskind-Glogower [29] phase operator and are defined in terms of the number states $|n\rangle$ as

$$
|\theta\rangle=\sum_{n=0}^{\infty} e^{i n \theta}|n\rangle .
$$

The sum in Eq. (32) is assumed to converge and the phase distribution normalized to unity. Now we take up two physically interesting initial conditions for the system $S$. 


\section{System initially in a coherent state}

The initial density matrix of the system is

$$
\rho^{s}(0)=|\alpha\rangle\langle\alpha|,
$$

where

$$
\alpha=|\alpha| e^{i \theta_{0}}
$$

is a coherent state [57]. Using Eqs. (31), (34) in Eq. (2) and then using it in Eq. (32), the phase distribution is obtained as [36]

$$
\begin{aligned}
\mathcal{P}(\theta) & =\frac{1}{2 \pi} \sum_{m, n=0}^{\infty} \frac{|\alpha|^{n+m}}{\sqrt{(n) !(m) !}} e^{i(n-m)\left(\theta-\theta_{0}\right)} e^{-|\alpha|^{2}} \\
& \times e^{-i \omega(m-n) t} e^{i(\hbar \omega)^{2}(m-n)(n+m+1) \eta(t)} e^{-(\hbar \omega)^{2}(n-m)^{2} \gamma(t)}
\end{aligned}
$$

\section{System initially in a squeezed coherent state}

The initial density matrix of the system is

$$
\rho^{s}(0)=|\xi, \alpha\rangle\langle\alpha, \xi|,
$$

where the squeezed coherent state is defined as [57]

$$
|\xi, \alpha\rangle=S(\xi) D(\alpha)|0\rangle .
$$

Here $S$ denotes the standard squeezing operator and $D$ denotes the standard displacement operator [57]. Using Eqs. (31), (37) in Eq. (21) and then using it in Eq. (32), the phase distribution is obtained as 36]

$$
\begin{aligned}
\mathcal{P}(\theta)= & \frac{1}{2 \pi} \sum_{m, n=0}^{\infty} e^{i(n-m) \theta} \frac{e^{i \frac{\psi}{2}(m-n)}}{2^{\frac{(m+n)}{2}} \sqrt{(m) !(n !)}} \frac{\left(\tanh \left(r_{1}\right)\right)^{\frac{(m+n)}{2}}}{\cosh \left(r_{1}\right)} \\
& \times \exp \left[-|\alpha|^{2}\left(1-\tanh \left(r_{1}\right) \cos \left(2 \theta_{0}-\psi\right)\right)\right] \\
& \times H_{m}\left[\frac{|\alpha| e^{i\left(\theta_{0}-\frac{\psi}{2}\right)}}{\sqrt{\sinh \left(2 r_{1}\right)}}\right] H_{n}^{*}\left[\frac{|\alpha| e^{i\left(\theta_{0}-\frac{\psi}{2}\right)}}{\sqrt{\sinh \left(2 r_{1}\right)}}\right] \\
& \times e^{-i \omega(m-n) t} e^{i(\hbar \omega)^{2}(m-n)(n+m+1) \eta(t)} e^{-(\hbar \omega)^{2}(n-m)^{2} \gamma(t)} .
\end{aligned}
$$

Here the system squeezing parameter $\xi=r_{1} e^{i \psi}$ and $H_{n}[z]$ is a Hermite polynomial. The phase distributions depicted by Eqs. (36), (39) have been plotted in Ref. [36], where they were seen to exhibit a phase diffusion pattern with the phase distributions being normailzed to unity.

\section{QUANTUM PHASE DISTRIBUTION OF A TWO-LEVEL ATOMIC SYSTEM IN NON-QND INTERACTION WITH BATH}

Here we will obtain the quantum phase distribution of a two-level atomic system in an interaction with a squeezed thermal bath such that it undergoes both decoherence and dissipation. The reduced density matrix operator of the system $S$ is given by [7, 57]

$$
\begin{aligned}
\frac{d}{d t} \rho^{s}(t) & =-i \frac{\omega}{2}\left[\sigma_{z}, \rho^{s}(t)\right] \\
& +\gamma_{0}(N+1)\left(\sigma_{-} \rho^{s}(t) \sigma_{+}-\frac{1}{2} \sigma_{+} \sigma_{-} \rho^{s}(t)-\frac{1}{2} \rho^{s}(t) \sigma_{+} \sigma_{-}\right) \\
& +\gamma_{0} N\left(\sigma_{+} \rho^{s}(t) \sigma_{-}-\frac{1}{2} \sigma_{-} \sigma_{+} \rho^{s}(t)-\frac{1}{2} \rho^{s}(t) \sigma_{-} \sigma_{+}\right) \\
& -\gamma_{0} M \sigma_{+} \rho^{s}(t) \sigma_{+}-\gamma_{0} M^{*} \sigma_{-} \rho^{s}(t) \sigma_{-}
\end{aligned}
$$


In the context of quantum information, the open system effect depicted by Eq. (40) can be modeled by a familiar noisy channel called the generalized amplitude damping channel [40, 43, 44] for zero bath squeezing. For the case of finite bath squeezing and temperature, the corresponding noisy channel has been obtained by us recently [58] and could appropriately be called the squeezed generalized amplitude damping channel.

In Eq. (40), $\gamma_{0}$, having the dimension of $(\text { time })^{-1}$, is the spontaneous emission rate given by

$$
\gamma_{0}=\frac{4 \omega^{3}|\vec{d}|^{2}}{3 \hbar c^{3}}
$$

and $\sigma_{+}, \sigma_{-}$are the standard raising and lowering operators, respectively given by

$$
\begin{aligned}
& \sigma_{+}=|1\rangle\langle 0|=\frac{1}{2}\left(\sigma_{x}+i \sigma_{y}\right), \\
& \sigma_{-}=|0\rangle\langle 1|=\frac{1}{2}\left(\sigma_{x}-i \sigma_{y}\right),
\end{aligned}
$$

with $\sigma_{z}$ being the standard Pauli operator related to the raising and lowering operators as $\left[\sigma_{+}, \sigma_{-}\right]=\sigma_{z}$. In the above equations, $[a, b]=a b-b a$. In Eq. (40)

$$
\begin{gathered}
N=N_{\mathrm{th}}\left(\cosh ^{2}(r)+\sinh ^{2}(r)\right)+\sinh ^{2}(r), \\
M=-\frac{1}{2} \sinh (2 r) e^{i \Phi}\left(2 N_{\mathrm{th}}+1\right) \equiv R e^{i \Phi},
\end{gathered}
$$

and

$$
N_{\mathrm{th}}=\frac{1}{e^{\frac{\hbar \omega}{k^{T}}}-1}
$$

Here $N_{\text {th }}$ is the Planck distribution giving the number of thermal photons at the frequency $\omega$ and $r$, $\Phi$ are squeezing parameters. The analogous case of a thermal bath without squeezing can be obtained from the above expressions by setting these squeezing parameters to zero. Eq. (40) can be solved using the Bloch vector formalism (cf. [7], [40]). However, the solutions obtained thus are not amenable to treatment of the quantum phase distribution by use of Eq. (17). For this purpose we briefly detail the solution of Eq. (40) in an operator form. We closely follow the derivation given by Nakazato et al. [59] and extend it to the case of a squeezed thermal bath.

The Eq. (40) can be written as

$$
\frac{d}{d t} \rho^{s}(t)=A \rho^{s}(t)+\rho^{s}(t) A^{\dagger}+\left[\gamma_{+} \sigma_{-} \rho^{s}(t) \sigma_{+}+\gamma_{-} \sigma_{+} \rho^{s}(t) \sigma_{-}-\gamma_{0} M \sigma_{+} \rho^{s}(t) \sigma_{+}-\gamma_{0} M^{*} \sigma_{-} \rho^{s}(t) \sigma_{-}\right]
$$

where

$$
\gamma_{+}=\gamma_{0}(N+1), \gamma_{-}=\gamma_{0} N
$$

and

$$
\begin{aligned}
A & =-\frac{1}{4} \gamma^{\beta}-\frac{1}{4}(\gamma+2 i \omega) \sigma_{z} \\
\gamma^{\beta} & =\gamma_{+}+\gamma_{-}=\gamma_{0}(2 N+1) \\
\gamma & =\gamma_{+}-\gamma_{-}=\gamma_{0}
\end{aligned}
$$

The following transformation is now introduced in Eq. (46):

$$
\rho^{s}(t)=e^{A t} \rho^{I}(t) e^{A^{\dagger} t}
$$

yielding

$$
\frac{d}{d t} \rho^{I}(t)=\gamma_{+} \sigma_{-} \rho^{I}(t) \sigma_{+} e^{-\gamma t}+\gamma_{-} \sigma_{+} \rho^{I}(t) \sigma_{-} e^{\gamma t}-\gamma_{0} M \sigma_{+} \rho^{I}(t) \sigma_{+} e^{i 2 \omega t}-\gamma_{0} M^{*} \sigma_{-} \rho^{I}(t) \sigma_{-} e^{-i 2 \omega t}
$$

The solution of Eq. (50) is facilitated by the introduction of superoperators having the following action:

$$
\begin{aligned}
& \mathcal{P}_{-} \rho=\sigma_{-} \rho \sigma_{+}, \mathcal{P}_{+} \rho=\sigma_{+} \rho \sigma_{-}, \\
& \mathcal{P}_{-}^{a} \rho=\sigma_{-} \rho \sigma_{-}, \mathcal{P}_{+}^{a} \rho=\sigma_{+} \rho \sigma_{+} .
\end{aligned}
$$


Using Eqs. (51), Eq. (50) can be written as

$$
\begin{aligned}
\frac{d}{d t} \rho^{I}(t) & =\left[\gamma_{+} e^{-\gamma t} \mathcal{P}_{-}+\gamma_{-} e^{\gamma t} \mathcal{P}_{+}\right] \rho^{I}(t) \\
& -\left[\gamma_{0} M e^{i 2 \omega t} \mathcal{P}_{+}^{a}+\gamma_{0} M^{*} e^{-i 2 \omega t} \mathcal{P}_{-}^{a}\right] \rho^{I}(t)
\end{aligned}
$$

Integrating we get

$$
\begin{aligned}
\rho^{I}(t) & =\rho^{I}(0)+\frac{1}{\gamma^{\beta}}\left[\gamma_{+}\left(e^{\gamma_{-} t}-e^{-\gamma_{+} t}\right) \mathcal{P}_{-}+\left(\gamma_{+} e^{\gamma_{-} t}+\gamma_{-} e^{-\gamma_{+} t}-\gamma^{\beta}\right) \mathcal{P}_{-} \mathcal{P}_{+}\right] \rho^{I}(0) \\
& +\frac{1}{\gamma^{\beta}}\left[\gamma_{-}\left(e^{\gamma_{+} t}-e^{-\gamma_{-} t}\right) \mathcal{P}_{+}+\left(\gamma_{-} e^{\gamma_{+} t}+\gamma_{+} e^{-\gamma_{-} t}-\gamma^{\beta}\right) \mathcal{P}_{+} \mathcal{P}_{-}\right] \rho^{I}(0) \\
& -\gamma_{0} M\left[\frac{\sinh (\alpha t)}{\alpha} e^{i \omega t} \mathcal{P}_{+}^{a}-\frac{1}{\gamma_{0} M}\left(e^{i \omega t}\left\{\cosh (\alpha t)-\frac{i \omega}{\alpha} \sinh (\alpha t)\right\}-1\right) \mathcal{P}_{+}^{a} \mathcal{P}_{-}^{a}\right] \rho^{I}(0) \\
& -\gamma_{0} M^{*}\left[\frac{\sinh (\alpha t)}{\alpha} e^{-i \omega t} \mathcal{P}_{-}^{a}-\frac{1}{\gamma_{0} M^{*}}\left(e^{-i \omega t}\left\{\cosh (\alpha t)+\frac{i \omega}{\alpha} \sinh (\alpha t)\right\}-1\right) \mathcal{P}_{-}^{a} \mathcal{P}_{+}^{a}\right] \rho^{I}(0),
\end{aligned}
$$

where

$$
\alpha=\sqrt{\gamma_{0}^{2}|M|^{2}-\omega^{2}} .
$$

All the other terms are as given above. Using Eq. (49) in Eq. (53) we finally obtain the solution of Eq. (40) as

$$
\begin{aligned}
\rho^{s}(t) & =\frac{1}{4} \rho^{s}(0)\left(1+e^{-\gamma^{\beta} t}+2 \cosh (\alpha t) e^{-\frac{\gamma^{\beta} t}{2}}\right) \\
& +\frac{1}{4} \sigma_{z} \rho^{s}(0) \sigma_{z}\left(1+e^{-\gamma^{\beta} t}-2 \cosh (\alpha t) e^{-\frac{\gamma^{\beta} t}{2}}\right) \\
& -\frac{1}{4} \rho^{s}(0) \sigma_{z}\left(\frac{\gamma}{\gamma^{\beta}}\left(1-e^{-\gamma^{\beta} t}\right)-\frac{2 i \omega}{\alpha} \sinh (\alpha t) e^{-\frac{\gamma^{\beta} t}{2}}\right) \\
& -\frac{1}{4} \sigma_{z} \rho^{s}(0)\left(\frac{\gamma}{\gamma^{\beta}}\left(1-e^{-\gamma^{\beta} t}\right)+\frac{2 i \omega}{\alpha} \sinh (\alpha t) e^{-\frac{\gamma^{\beta} t}{2}}\right) \\
& +\left(1-e^{-\gamma^{\beta} t}\right)\left(\frac{\gamma_{+}}{\gamma^{\beta}} \sigma_{-} \rho^{s}(0) \sigma_{+}+\frac{\gamma_{-}}{\gamma^{\beta}} \sigma_{+} \rho^{s}(0) \sigma_{-}\right) \\
& -\gamma_{0} \frac{\sinh (\alpha t)}{\alpha} e^{-\frac{\gamma^{\beta} t}{2}}\left(M \sigma_{+} \rho^{s}(0) \sigma_{+}+M^{*} \sigma_{-} \rho^{s}(0) \sigma_{-}\right) .
\end{aligned}
$$

This is the desired form of solution of the master equation (40). For the case of a thermal bath without squeezing, $r$ and $\Phi$ are zero and it can be seen that Eq. (55) reduces to the solution obtained by Nakazato et al. [59] for the case of a two-level atom interacting with a thermal bath. We will use Eq. (55) in the following subsections to investigate the quantum phase distribution.

\section{A. System initially in an atomic coherent state}

Taking the intial density matrix of the system $S$ to be as in Eq. (18), using it in Eq. (55), and then in Eq. (17), with $j=\frac{1}{2}$, we obtain the quantum phase distribution as

$$
\begin{aligned}
\mathcal{P}(\phi)= & \frac{1}{2 \pi}\left[1+\frac{\pi}{4 \alpha} \sin \left(\alpha^{\prime}\right)\left\{\alpha \cosh (\alpha t) \cos \left(\phi-\beta^{\prime}\right)+\omega \sinh (\alpha t) \sin \left(\phi-\beta^{\prime}\right)\right.\right. \\
& \left.\left.-\gamma_{0} R \sinh (\alpha t) \cos \left(\Phi+\beta^{\prime}+\phi\right)\right\} e^{-\frac{\gamma^{\beta} t}{2}}\right]
\end{aligned}
$$

Here $R, \Phi$ come from Eq. (44), and $\gamma^{\beta}, \alpha$ are as in Eqs. (48) and (54), respectively. The Eq. (56) can be seen to be normalized to unity. When $\gamma_{0}$ is set equal to zero, i.e., for the case where the effects of the bath are neglected, Eq. (56) becomes

$$
\mathcal{P}\left(\phi, \gamma_{0}=0\right)=\frac{1}{2 \pi}\left\{1+\frac{\pi}{4} \sin \left(\alpha^{\prime}\right) \cos \left(\beta^{\prime}+\omega t-\phi\right)\right\}
$$




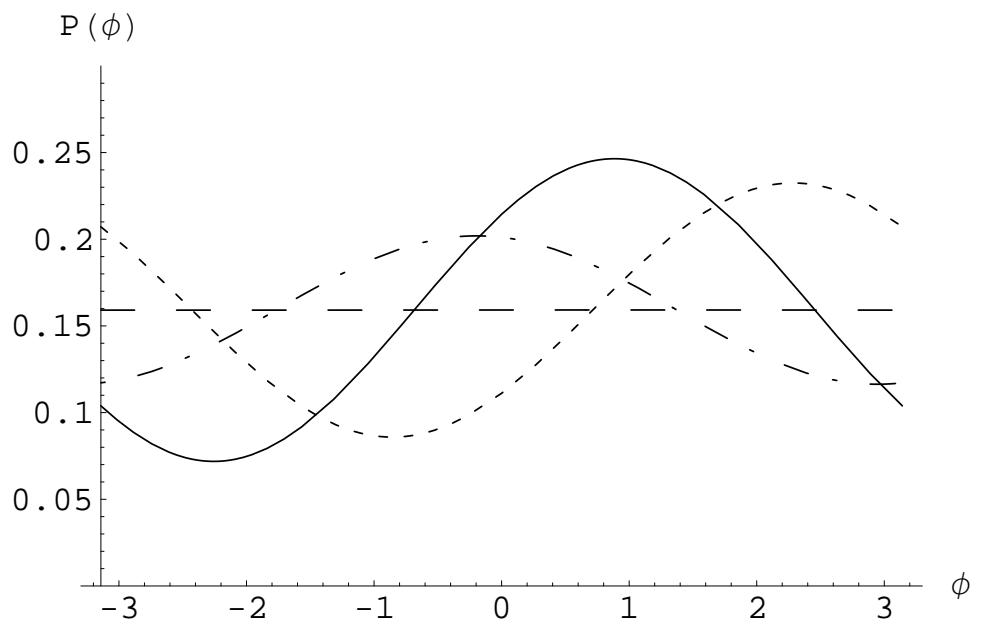

FIG. 2: Quantum phase distribution $\mathcal{P}(\phi)$ (Eq. (56) ) with respect to $\phi$ (in radians), for a two-level dissipative system initially in an atomic coherent state (18). Here $\omega=1.0, \Phi=\pi / 8, \alpha^{\prime}=\beta^{\prime}=\pi / 4$, and $\gamma_{0}=0.25$. The bold and small-dashed curves correspond to temperature (in units where $\hbar \equiv k_{B} \equiv 1$ ) $T=0$, bath squeezing parameter $r=0$, but with bath exposure times $t=0.1$ and 1.5, respectively. The large-dashed and dot-dashed curves correspond to $T=300$ and $t=0.1$, but $r=0$ and 2 , respectively. Comparing the last two curves, we note that, counterintuitively, squeezing resists diffusion.

In the analogous case of the QND system-bath interaction, the phase distribution was given by Eq. (19) which with the bath coupling parameter $\gamma_{0}$ set to zero, is easily seen to reduce to Eq. (57). This is a nice consistency check for these equations.

Figure2 2 illustrates the combined effects of temperature, evolution time and bath squeezing $(r, \Phi)$ on quantum phase distribution. Comparison of the small- and large-dashed curves brings out the diffusive influence of temperature, while a comparison of the bold and small-dashed curves shows that the phase distribution shifts with increase in bath exposure time. On the other hand, a comparison between the large- and dot-dashed curves illustrates an interesting feature of squeezing in dissipative systems governed by Lindblad-type equations (40), in that squeezing tends to counteract the influence of temperature, which in this case manifests as resistence to randomization of phase. A similar behavior is observed in the joint effect of temperature and squeezing on the geometric phase of a qubit (two-level system) interacting dissipatively with its environment [40]. The normalization of the phase distribution is preserved.

We plot in Figure 3 the function

$$
\begin{aligned}
p(m=1 / 2, t) & =\left\langle 1 / 2\left|\rho^{s}(t)\right| 1 / 2\right\rangle \\
& =\frac{1}{2}\left[\left(1-\frac{\gamma_{0}}{\gamma^{\beta}}\right)+\left(1+\frac{\gamma_{0}}{\gamma^{\beta}}\right) e^{-\gamma^{\beta} t}\right] \sin ^{2}\left(\alpha^{\prime} / 2\right)+\frac{\gamma_{-}}{\gamma^{\beta}}\left(1-e^{-\gamma^{\beta} t}\right) \cos ^{2}\left(\alpha^{\prime} / 2\right) .
\end{aligned}
$$

Figure 3 depicts an expected behavior of a two-level system subjected to a dissipative channel. In particular, for $T=0$ and $r=0$, it drives the system towards a pure state (with $m=-1 / 2$ ) and thus behaves as a quantum deleter [43]. Correspondingly, the phase distribution $P(\phi)$ tends to level out for large bath exposure time $t$, as seen in Figure 4. This brings out nicely the complementarity between $p(m)$ and $P(\phi)$ [1]. It is to be noted that, in contrast to the QND case, here the Wigner-Dicke states are not the preferred basis, and hence the environmental effects manifest themselves in the function $p(m)$ as seen in Eq. (58).

\section{B. System initially in an atomic squeezed state}

Taking the intial density matrix of the system $S$ to be as in Eq. (22), using it in Eq. (55), and then in Eq. (17), with $j=\frac{1}{2}$, we obtain the quantum phase distribution for $p= \pm \frac{1}{2}$ as

$$
\mathcal{P}(\phi)=\frac{1}{2 \pi}\left[1 \pm \frac{\pi}{4 \cosh (\Theta)}\left\{\cosh (\alpha t) \cos (\phi)+\frac{\omega}{\alpha} \sinh (\alpha t) \sin (\phi)-\frac{\gamma_{0} R}{\alpha} \sinh (\alpha t) \cos (\phi+\Phi)\right\} e^{-\frac{\gamma^{\beta_{t}}}{2}}\right] .
$$

Here $\Theta$ is as defined in Eq. (21) and all the other terms are as given above. The Eqs. (59) are easily seen to be normalized to unity. Also by setting $\gamma_{0}$ to zero in them, they are seen to reduce to the cases of $\gamma_{0}$ set to zero in their 


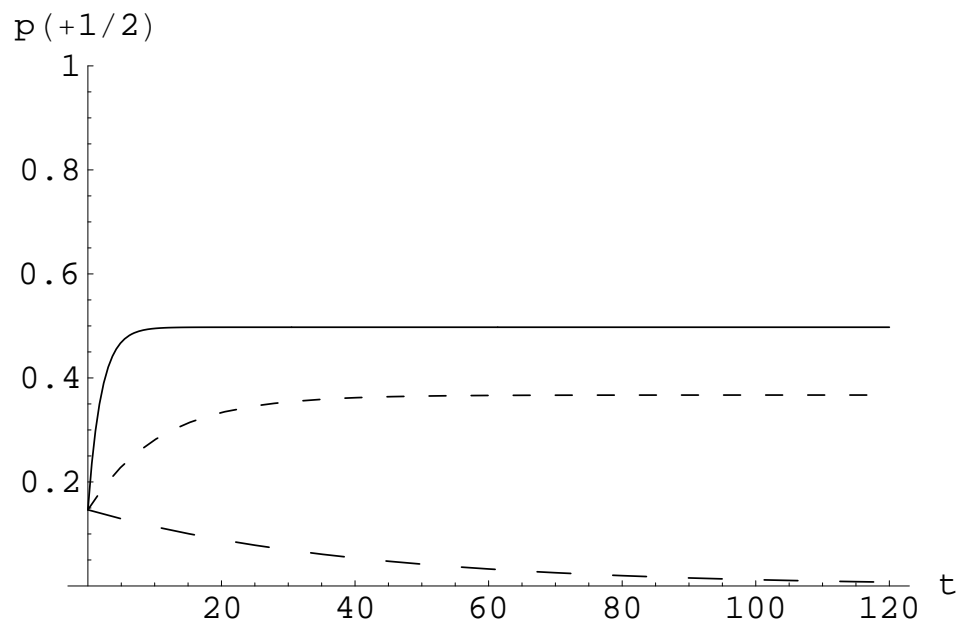

FIG. 3: The distribution $p(m=1 / 2, t)$ (Eq. (58) ) for a two-level dissipative system starting in an atomic coherent state (Eq. (18), as a function of time for different environmental conditions. The bold curve corresponds to temperature $T=100$, $\gamma_{0}=0.0025, r=\Phi=0, \omega=1, \alpha^{\prime}=\beta^{\prime}=\pi / 4$, illustrative of a system becoming maximally mixed with time. The large-dashed curve corresponds to $T=0, \gamma_{0}=0.025, r=0$, and depicts quantum deletion [43]. The small-dashed curve represents the case $T=0, \gamma_{0}=0.025, r=1$. Here time and temperature are in units where $\hbar \equiv k_{B} \equiv 1$.

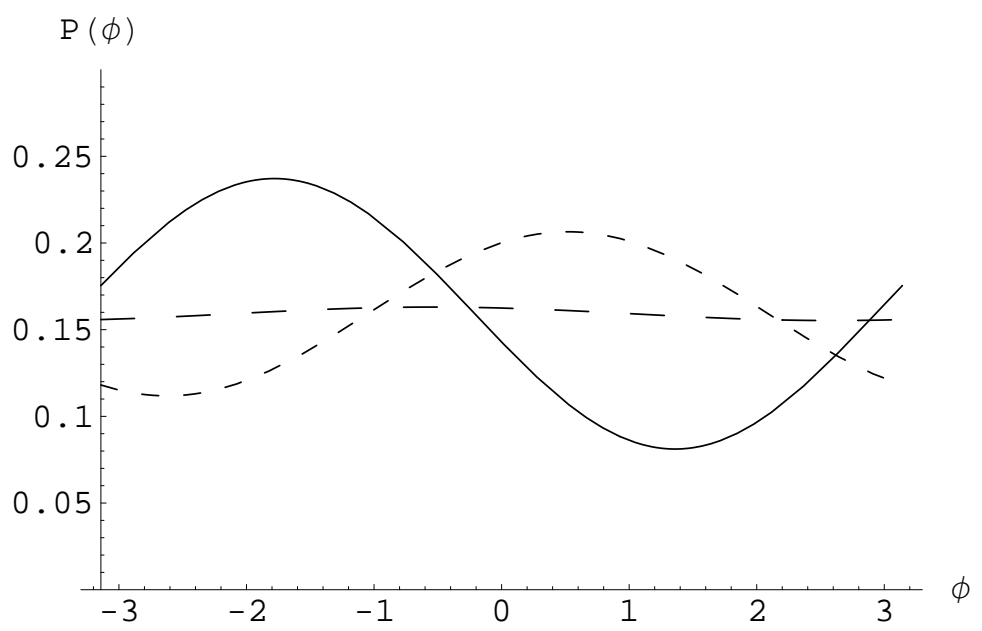

FIG. 4: Quantum phase distribution $\mathcal{P}(\phi)$ (Eq. (56)) with respect to $\phi$ (in radians), for a two-level dissipative system starting in an atomic coherent state (Eq. (18), at various times with temperature (in units where $\hbar \equiv k_{B} \equiv 1$ ) $T=0$ and bath squeezing parameters $r=\Phi=0, \gamma_{0}=0.025, \omega=1, \alpha^{\prime}=\beta^{\prime}=\pi / 4$. The large-dashed, small-dashed and bold curves correspond, respectively, to evolution times $t=250,50$ and 10. The large-dashed curve depicts the randomization of phase distribution at long times. Comparison of this figure with Figure 3 clearly brings out complementarity between the 'number' and 'phase' variables. In particular, comparision between the large-dashed curves in both Figures shows how as the state becomes increasing pure, tending to $m=-1 / 2$, with time, the corresponding complementary distribution $P(\phi)$ level outs.

QND counterparts, Eqs. (23), respectively. This serves as a consistency check for these equations. On comparing the above equations, for the quantum phase distributions, with the corresponding ones for the case of QND system-bath interaction, these are easily seen to be more complicated. This is a reflection of the fact that the phase distributions developed in this section are for a process that involves both dephasing as well as dissipation, in contrast to the QND case, which involves only dephasing.

We plot in Figures 5 the quantum phase distributions $\mathcal{P}(\theta)$ for a two-level system starting in an atomic squeezed state (22). An interesting feature in Figures 5 is brought out by a comparison of the bold and large-dashed curves. Squeezing is seen to have the effect of resisting the diffusive effect of temperature on the phase. This is similar to the behavior seen in Figure 2 and suggests that this is a generic property of squeezing in a dissipative interaction. A comparison of the small-dashed and bold curves brings out the diffusive effect of temperature on the phase distribution while a comparison between the small-dashed and dot-dashed curves shows that the distribution shifts with time. 


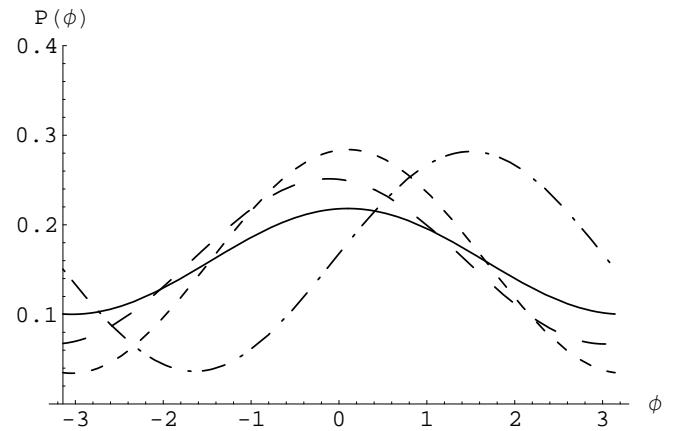

(a)

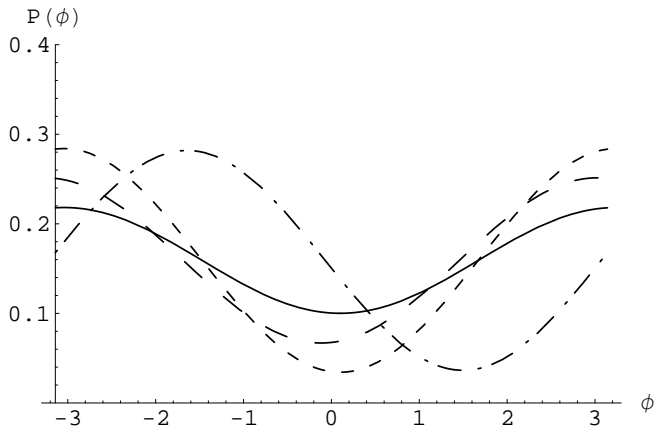

(b)

FIG. 5: Quantum phase distribution $\mathcal{P}(\phi)$ (Eq. (59) with respect to $\phi$ (in radians) for a two-level system starting in an atomic squeezed state (22). Here $\omega=1.0, \Phi=\pi / 8, \Theta=-0.01832$ and $\gamma_{0}=0.025$. Figure (a) refers to $p=\frac{1}{2}$ and (b) to $p=-\frac{1}{2}$. In both figures, the large-dashed and bold curves correspond to temperature (in units where $\hbar \equiv k_{B} \equiv 1$ ) $T=300$ and evolution time $t=0.1$. The bath squeezing parameter $r$ is, respectively, 0.5 and 0.0 . The small-dashed and dot-dashed curves correspond to $T=0$ and $r=0.0$, with time $t$ being 0.1 and 1.5 , respectively.

The phase distribution normalization is preserved.

\section{QUANTUM PHASE DISTRIBUTION OF A HARMONIC-OSCILLATOR SYSTEM IN NON-QND INTERACTION WITH BATH}

Here we will obtain the quantum phase distribution of a harmonic-oscillator system, $H_{s}=\hbar \omega\left(a^{\dagger} a+\frac{1}{2}\right)$, in a dissipative interaction with a squeezed thermal bath. The reduced density matrix operator of the system $S$, in the interaction picture, is given by [7, 57]

$$
\begin{aligned}
\frac{d}{d t} \rho^{s}(t) & =\gamma_{0}(N+1)\left(a \rho^{s}(t) a^{\dagger}-\frac{1}{2} a^{\dagger} a \rho^{s}(t)-\frac{1}{2} \rho^{s}(t) a^{\dagger} a\right) \\
& +\gamma_{0} N\left(a^{\dagger} \rho^{s}(t) a-\frac{1}{2} a a^{\dagger} \rho^{s}(t)-\frac{1}{2} \rho^{s}(t) a a^{\dagger}\right) \\
& +\gamma_{0} M\left(a^{\dagger} \rho^{s}(t) a^{\dagger}-\frac{1}{2}\left(a^{\dagger}\right)^{2} \rho^{s}(t)-\frac{1}{2} \rho^{s}(t)\left(a^{\dagger}\right)^{2}\right) \\
& +\gamma_{0} M^{*}\left(a \rho^{s}(t) a-\frac{1}{2}(a)^{2} \rho^{s}(t)-\frac{1}{2} \rho^{s}(t)(a)^{2}\right) .
\end{aligned}
$$

In the above equation, $N, M$ are bath parameters which will be given below and $\gamma_{0}$ is a parameter which depends upon the system-bath coupling strength. The Eq. (60) can be solved using a variety of methods (cf. [7], [57]). However, the solutions obtained thus are not amenable to treatment of the quantum phase distribution by use of Eq. (32). For this purpose we again briefly detail the solution of Eq. (60) in an operator form. We closely follow the derivation given by $\mathrm{Lu}$ et al. 60]. The following transformations are introduced [61]:

$$
\rho^{\prime} s(t)=S^{\dagger}(\zeta) \rho^{s}(t) S(\zeta), a^{\prime}=S^{\dagger}(\zeta) a S(\zeta),
$$

where

$$
S(\zeta)=e^{\frac{1}{2}\left(\zeta^{*} a^{2}-\zeta a^{\dagger 2}\right)}
$$

Using Eqs. (61) we get

$$
a^{\prime}=\cosh (|\zeta|) a-\frac{\zeta}{|\zeta|} \sinh (|\zeta|) a^{\dagger}
$$

Using Eqs. (61), (63) in Eq. (60), we get

$$
\frac{d}{d t} \rho^{\prime s}(t)=\left[\alpha K_{+}+\beta K_{-}+(\alpha+\beta) K_{0}+\frac{\gamma_{0}}{2}\right] \rho^{\prime s}(t),
$$


where

$$
\begin{gathered}
\alpha=\gamma_{0} N \cosh (2|\zeta|)+\gamma_{0} \cosh ^{2}(|\zeta|)-\frac{\gamma_{0}}{2|\zeta|} \sinh (2|\zeta|)\left(M \zeta^{*}+M^{*} \zeta\right), \\
\beta=\gamma_{0} N \cosh (2|\zeta|)+\gamma_{0} \sinh ^{2}(|\zeta|)-\frac{\gamma_{0}}{2|\zeta|} \sinh (2|\zeta|)\left(M \zeta^{*}+M^{*} \zeta\right) .
\end{gathered}
$$

The parameters involved in the above equation need to satisfy the following consistency condition:

$$
\frac{|\zeta|}{\zeta} M \operatorname{coth}(|\zeta|)+\frac{\zeta}{|\zeta|} M^{*} \tanh (|\zeta|)=2 N+1
$$

It can be seen that

$$
\begin{aligned}
M & =\frac{1}{2} \sinh (2 r)\left(2 N_{\mathrm{th}}+1\right) e^{i \Phi}, \\
N & =N_{\mathrm{th}}\left(\cosh ^{2}(r)+\sinh ^{2}(r)\right)+\sinh ^{2}(r), \\
N_{\mathrm{th}} & =\frac{1}{e^{\frac{\hbar \omega}{k_{B} T}}-1}, \zeta=r e^{i \Phi},
\end{aligned}
$$

satisfy Eq. (66). In Eq. (64), $K_{+}, K_{-}$and $K_{0}$ are superoperators satisfying

$$
K_{+} \rho^{\prime} s=a \rho^{\prime} s a^{\dagger}, K_{-} \rho^{\prime s}=a^{\dagger} \rho^{\prime} s a, K_{0} \rho^{\prime} s=-\frac{1}{2}\left(a^{\dagger} a \rho^{\prime} s+\rho^{\prime} s a^{\dagger} a+\rho^{\prime} s\right) .
$$

These superoperators can be seen to satisfy:

$$
\left[K_{-}, K_{+}\right] \rho^{\prime s}=2 K_{0} \rho^{\prime} s,\left[K_{0}, K_{ \pm}\right] \rho^{\prime} s= \pm K_{ \pm} \rho^{\prime} s,
$$

which coincides with the commutation relations of the $s u(1,1)$ Lie algebra. This brings out the intimate connection between the solutions of the master equation (60) and the generators of the $s u(1,1)$ Lie algebra. Using the disentangling theorems of the $s u(1,1)$ Lie algebra, Eq. (64) can be solved to yield:

$$
\rho^{\prime} s(t)=e^{\frac{\gamma_{0} t}{2}} e^{y_{-}(t) K_{-}} e^{\ln \left(y_{0}(t)\right) K_{0}} e^{y_{+}(t) K_{+}} \rho^{\prime s}(0),
$$

where

$$
\begin{aligned}
& y_{0}(t)=\left(\frac{\alpha e^{\frac{\gamma_{0} t}{2}}-\beta e^{-\frac{\gamma_{0} t}{2}}}{\gamma_{0}}\right)^{2}, \\
& y_{+}(t)=\frac{\alpha\left(e^{-\gamma_{0} t}-1\right)}{\left(\beta e^{-\gamma_{0} t}-\alpha\right)} \\
& y_{-}(t)=\frac{\beta\left(e^{-\gamma_{0} t}-1\right)}{\left(\beta e^{-\gamma_{0} t}-\alpha\right)} .
\end{aligned}
$$

Using Eqs. (70), (61), the solution of Eq. (60) can be written as

$$
\rho^{s}(t)=S(\zeta)\left\{e^{\frac{\gamma_{0} t}{2}} e^{y_{-}(t) K_{-}} e^{\ln \left(y_{0}(t)\right) K_{0}} e^{y_{+}(t) K_{+}} S^{\dagger}(\zeta) \rho^{s}(0) S(\zeta)\right\} S^{\dagger}(\zeta) .
$$

This is the form of solution of the master equation which we will use for investigation of the quantum phase distribution. We will use a special initial state of the system, the squeezed coherent state,

$$
\rho^{s}(0)=|\zeta, \eta\rangle\langle\eta, \zeta|
$$

where

$$
|\zeta, \eta\rangle=S(\zeta) D(\eta)|0\rangle
$$

Here $|0\rangle$ is the vacuum state and $D(\eta)$ is the standard displacement operator. Substituting Eq. (73) in Eq. (72), the solution of the Eq. (60) starting from the initial state (73), following Lu et al. [60], is obtained as

$$
\begin{aligned}
\rho^{s}(t)= & \frac{1}{(1+\tilde{\beta}(t))} e^{-\tilde{\beta}(t)|\tilde{\eta}(t)|^{2}} \sum_{k=0}^{\infty}\left(\frac{\tilde{\beta}(t)}{(1+\tilde{\beta}(t))}\right)^{k} \frac{1}{k !} \times \\
& \sum_{l, p=0}^{k}\left(\begin{array}{c}
k \\
l
\end{array}\right)\left(\begin{array}{c}
k \\
p
\end{array}\right) \sqrt{l ! p !}\left(\tilde{\eta}^{*}(t)\right)^{k-l}(\tilde{\eta}(t))^{k-p}|\zeta, \tilde{\eta}(t), l\rangle\langle p, \tilde{\eta}(t), \zeta|,
\end{aligned}
$$


where

$$
|\zeta, \tilde{\eta}(t), l\rangle=S(\zeta)|\tilde{\eta}(t), l\rangle=S(\zeta) D(\tilde{\eta}(t))|l\rangle,
$$

and

$$
\tilde{\beta}(t)=\frac{\beta}{\gamma_{0}}\left(1-e^{-\gamma_{0} t}\right), \tilde{\eta}(t)=\eta \frac{e^{-\frac{\gamma_{0} t}{2}}}{(1+\tilde{\beta}(t))},
$$

where $\beta$ is given by Eq. (65). In Eq. (76), $D(\tilde{\eta}(t))=e^{\tilde{\eta}(t) a^{\dagger}-\tilde{\eta}^{*}(t) a}$ and $D(\tilde{\eta}(t))|l\rangle$ is known as the generalized coherent state (GCS) [62, 63] and thus the state $|\zeta, \tilde{\eta}(t), l\rangle$ would be the generalized squeezed coherent state (GSCS) [63]. The GCS's were introduced by Roy and Singh [62], where they demonstrated that the harmonic oscillator possesses an infinite string of coherent states. We see from Eqs. (75) and (73) that under the action of the master equation (60), which is of a Lindblad kind, a harmonic oscillator starting in a squeezed coherent state ends in a mixture that can be expressed as a sum over GSCS. Thus the above case can be thought of as a concrete physical realization of GSCS.

This is an example of ultracoherence pertaining to master equations governing the Lindblad type of evolution such as Eq. (60). Ultracoherence refers to the structure induced into the Fock space $\mathcal{F}(\mathcal{H})$, over a finite or infinite dimensional Hilbert space $\mathcal{H}$, by the action of all canonical transformations, both homogeneous (e.g., squeezing operation) and inhomogeneous (Weyl operators) [64, 65]. Starting from the squeezed coherent state (73) of the harmonic oscillator, obtained by applying the canonical transformation $U=S(\zeta) D(\eta)$ (74) to the vacuum state, and applying a canonical transformation (61) to the master equation (60), results in a mixture of ultracoherent states, which in this case is the GSCS.

Making use of the Fock-space representation of GCS [62]

$$
|n, \alpha(t)\rangle=e^{-\frac{|\alpha(t)|^{2}}{2}} \sum_{l=0}^{\infty}\left(\frac{n !}{l !}\right)^{\frac{1}{2}} L_{n}^{l-n}\left(|\alpha(t)|^{2}\right)[\alpha(t)]^{l-n}|l\rangle,
$$

where $L_{n}^{l-n}(x)$ is the generalized Laguerre polynomial, and substituting Eq. (75) in Eq. (32), reverting back to the Schrödinger picture, we obtain the quantum phase distribution of a dissipative harmonic oscillator starting in a squeezed coherent state (73) as

$$
\begin{aligned}
\mathcal{P}(\theta) & =\frac{1}{2 \pi} e^{-|\tilde{\eta}(t)|^{2}} \frac{e^{-\tilde{\beta}(t)|\tilde{\eta}(t)|^{2}}}{(1+\tilde{\beta}(t))} \sum_{m, n} e^{-i \omega(m-n) t} e^{i(n-m) \theta} \sum_{u, v, k} G_{u, m}^{*}(\zeta) G_{v, n}(\zeta) \\
& \times\left(\frac{\tilde{\beta}(t)}{(1+\tilde{\beta}(t))}\right)^{k} \frac{1}{k !} \sum_{l, p=0}^{k}\left(\begin{array}{c}
k \\
l
\end{array}\right)\left(\begin{array}{c}
k \\
p
\end{array}\right) \frac{l ! p !}{\sqrt{(u ! v !)}}\left(\tilde{\eta}^{*}(t)\right)^{v-p+k-l}(\tilde{\eta}(t))^{u-l+k-p} \\
& \times L_{l}^{u-l}\left(|\tilde{\eta}(t)|^{2}\right) L_{p}^{* v-p}\left(|\tilde{\eta}(t)|^{2}\right) .
\end{aligned}
$$

In the above equation, $G_{m, n}(\zeta)=\langle m|S(\zeta)| n\rangle$ and is explicitly given, with $\zeta=r_{1} e^{i \phi}$, as [63]

$$
\begin{aligned}
G_{2 m, 2 p}= & \frac{(-1)^{p}}{(p) !(m) !}\left(\frac{(2 p) !(2 m) !}{\cosh \left(r_{1}\right)}\right)^{\frac{1}{2}} \exp (i(m-p) \phi) \\
& \times\left(\frac{\tanh \left(r_{1}\right)}{2}\right)^{(m+p)} F_{1}^{2}\left[-p,-m ; \frac{1}{2} ;-\frac{1}{\left(\sinh \left(r_{1}\right)\right)^{2}}\right] .
\end{aligned}
$$

Similarly $G_{2 m+1,2 p+1}(\zeta)$ is given by

$$
\begin{aligned}
G_{2 m+1,2 p+1}= & \frac{(-1)^{p}}{(p) !(m) !}\left(\frac{(2 p+1) !(2 m+1) !}{\cosh ^{3}\left(r_{1}\right)}\right)^{\frac{1}{2}} \exp (i(m-p) \phi) \\
& \times\left(\frac{\tanh \left(r_{1}\right)}{2}\right)^{(m+p)} F_{1}^{2}\left[-p,-m ; \frac{3}{2} ;-\frac{1}{\left(\sinh \left(r_{1}\right)\right)^{2}}\right] .
\end{aligned}
$$

As has been pointed out in [63], $G_{m, n}$ is nonzero only for either $m, n$ both even or both odd. For convenience it is sometimes assumed that $\phi$ is zero and $z=r_{1}$ is real. Here $r_{1}=r$, due to the initial condition (73) and $F_{1}^{2}$ is the Gauss hypergeometric function [66]. 


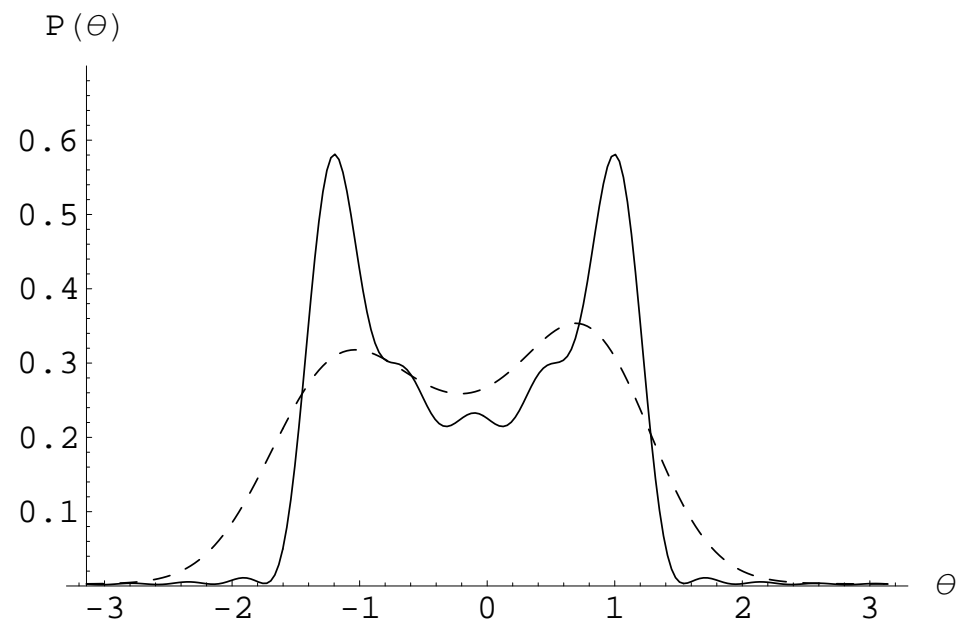

FIG. 6: A comparison of the quantum phase distributions $\mathcal{P}(\theta)$, for a harmonic oscillator system starting in a squeezed coherent state, for QND system-bath interaction (Eq. (39)) with that for dissipative system-bath interaction (Eq. (799)). The former (latter) is represented by the dashed (solid) curve. In both cases, temperature (in units where $\hbar \equiv k_{B} \equiv 1$ ) $T=0$, the squeezing parameters $r=r_{1}=1$, bath exposure time $t=0.1, \gamma_{0}=0.025, \omega=1$. In the former case, $\psi=0$ and $\omega_{c}=100$, while in the latter, $\Phi=0$.

In Figure 6. we make a comparison of the quantum phase distributions $\mathcal{P}(\theta)$ for a harmonic oscillator system starting in a squeezed coherent state (38), for QND system-bath interaction (Eq. (39)) with that for dissipative system-bath interaction (Eq. (79) ). A comparison of the distributions brings out the differing effects of the two types of system-bath interactions on them. The phase distributions are normalized.

\section{APPLICATIONS: PHASE DISPERSION}

From the perspective of experiments, a relevant quantity is the quantum phase fluctuation, which may be quantified by the variance $\sigma^{2}=\left\langle\phi^{2}\right\rangle-\langle\phi\rangle^{2}$. For example, Ref. [17] presents measurement of phase variance on atomic populations using interferometry improved by QND measurements at the inputs to the interferometer. However, this measure of phase fluctuation has the drawback that it depends on the origin of the phase integration. A measure of phase fluctuation that avoids this problem is the dispersion $D$ [27, 67, 68, 69],

$$
D=1-\left|\int_{-\pi}^{+\pi} d \phi e^{-i \phi} \mathcal{P}(\phi)\right|^{2}
$$

In this section, as an application of the phase distribution formalism employed above, we study the phase dispersion $D$ from these distributions. We also evaluated the variance for these distributions (not presented in this work), and found that in certain cases, there is in fact a qualitative difference of the behavior of these two quantities.

Figure 7 depicts the behavior of dispersion $D$ of $\phi$ of a ten two-level atomic system, starting from an atomic squeezed state, interacting with a squeezed thermal bath via a QND interaction, with respect to environmental squeezing parameter $r$ (Eq. (10)). The dispersion is found to increase with temperature and squeezing, tending to the maximal value of 1 , corresponding to the uniform distribution $P(\phi)=1 / 2 \pi$. This indicates that for a QND type system-bath interaction both temperature and squeezing have a similar effect of causing diffusion of the phase. Increasing bath exposure time $t$ also leads to the effect of leveling out $D$ to 1 . At $T=0$, this leveling out takes a much longer time on account of the logarithmic dependence of $\gamma(t)$ (Eq. (8)) on $t$, indicating a power-law decay.

Figure 8 is analogous to Figure 7 except that the dispersion of $\phi$ is plotted with respect to system squeezing parameter $\zeta$ (Eq. (21)). As $\Theta$ appearing in the expression for $P(\phi)$ has a logarithmic dependence on $\zeta$ (21), dispersion is insensitive to change in $\zeta$ over the plotted range. However, as expected, the dispersion increases with temperature because of the diffusive effect of temperature on the phase distribution.

Figure 9illustrates the behavior of dispersion $D$ of $\phi$ of a harmonic oscillator starting from a squeezed coherent state interacting with a squeezed thermal bath via a QND interaction, with respect to environmental squeezing parameter $r$ (Eq. (10)). The dispersion is found to increase with temperature and squeezing, tending to the maximal value 1. Here the large-dashed curve, which represents the case of unitary evolution, shows no variation with respect to change in environmental squeezing parameter $r$ (Eq. (10)), as expected. 


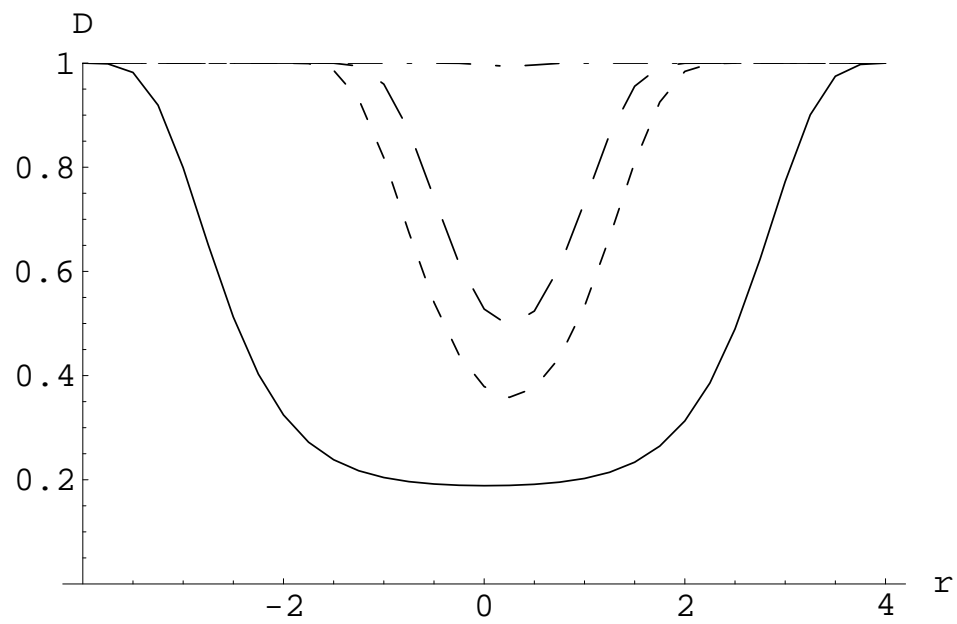

FIG. 7: Dispersion as function of environmental squeezing parameter $r$, for ten two-level atomic systems starting in an atomic squeezed state (Eq. (22)), at various temperatures for a QND system-environment interaction. Here $a=0.0$ [Eq. (10)], $\gamma_{0}=0.0025, \Theta=-0.01832, t=1.0, j=p=5, \omega=1.0$ and $\omega_{c}=100.0$. The bold, small-dashed, large-dashed and dot-dashed curves correspond to temperatures $T$ (in units where $\hbar \equiv k_{B}=1$ ) 0,50, 100 and 1000, respectively.

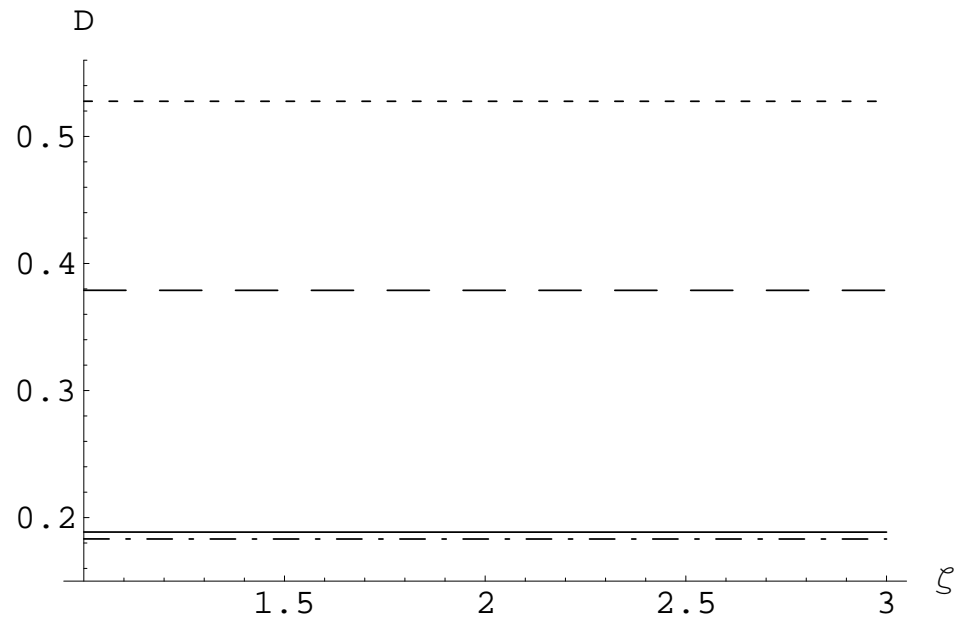

FIG. 8: Dispersion as function of system squeezing parameter $\zeta$, for ten two-level atomic systems starting in an atomic squeezed state (Eq. (22) ), at various temperatures for a QND system-environment interaction. Here $a=0.0, \gamma_{0}=0.0025, t=1.0$, $j=p=5, \omega=1.0, \omega_{c}=100.0$. The logarithmic dependence of $\Theta$ on $\zeta$ (Eq. (21)) implies a low sensitivity of the phase distribution to $\zeta$. The bold, dashed, dotted curves correspond to the temperatures (in units where $\hbar \equiv k_{B}=1$ ) $T=0.0,50.0$ and 100.0, respectively. The dot-dashed curve represents unitary evolution $\left(\gamma_{0}=0\right)$.

Figure 10 depicts the behavior of dispersion $D$ of $\phi$ of a two-level system starting in an atomic coherent state interacting with a squeezed thermal bath via a QND interaction, with respect to environmental squeezing parameter $r$ (Eq. (10)). As before, dispersion is found to level out with increase in temperature and squeezing, tending to the value 1 , which corresponds to a uniform distribution. We note that the pattern in this Figure is quite similar to that in Figure 7, whereas the use of variance for the data of Figure 10 produces a qualitatively different pattern.

Figure 11 shows the behavior of dispersion $D$ of $\phi$ of a two-level system starting in an atomic coherent state interacting with a squeezed thermal bath via a dissipative interaction, with respect to environmental squeezing parameter $r$ (Eq. (10)). While in the case of QND system-bath interaction (Figures 79 and 10), the dispersion is symmetric about $r=0$, it is not so in this case of dissipative interaction. Further, unlike in the case of QND interaction, here increase in absolute value of squeezing $(r)$ can cause a decrease in the dispersion. This illustrates the counteractive influence of the bath squeezing on the thermal diffusion of the phase distribution. This opposing behavior of temperature and squeezing seems to be generic to dissipative systems [40]. With increase in time $t$, phase tends to become randomized, increasing dispersion at any given squeezing towards the maximal value of 1 , indicative of the washing away of the non-stationary effects due to the squeezed bath [64]. From Figure 2, we see that increasing 


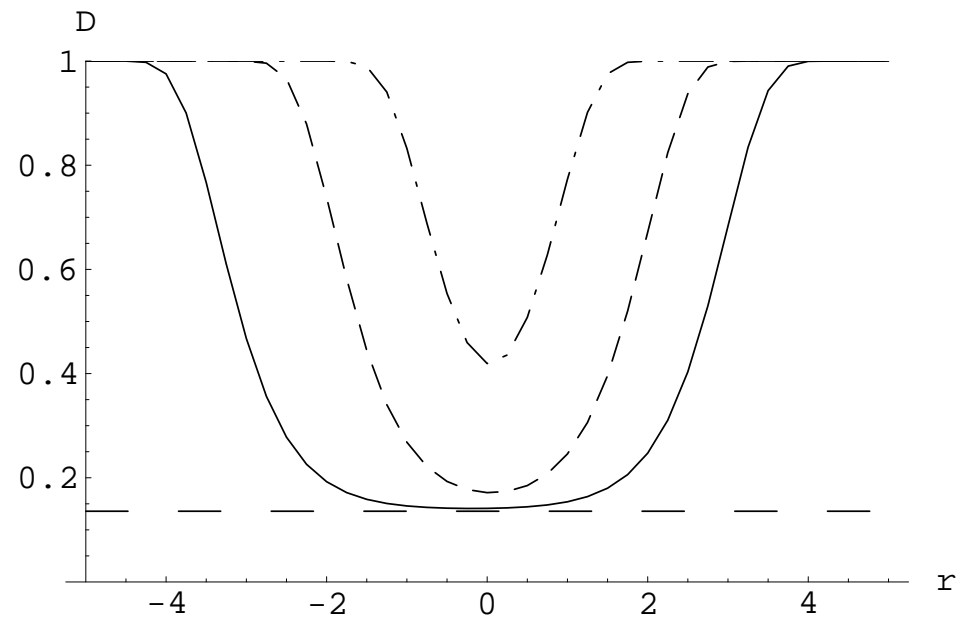

FIG. 9: Dispersion as function of environmental squeezing parameter $r$ for a harmonic oscillator starting in a squeezed coherent state (Eq. (37)) at various temperatures for a QND system-environment interaction. Here $\omega=1, \omega_{c}=100,|\alpha|^{2}=5$, $\gamma_{0}=0.0025$, and $t=0.1$. Here the parameter $a=0$, and the system squeezing parameters are $r_{1}=0.5$ and $\psi=\pi / 4$. The bold, small-dashed and dot-dashed curves correspond to temperatures (in units where $\hbar \equiv k_{B}=1$ ) $T=0,100$ and 1000 , respectively. The large-dashed curve corresponds to unitary evolution $\left(\gamma_{0}=0\right)$.

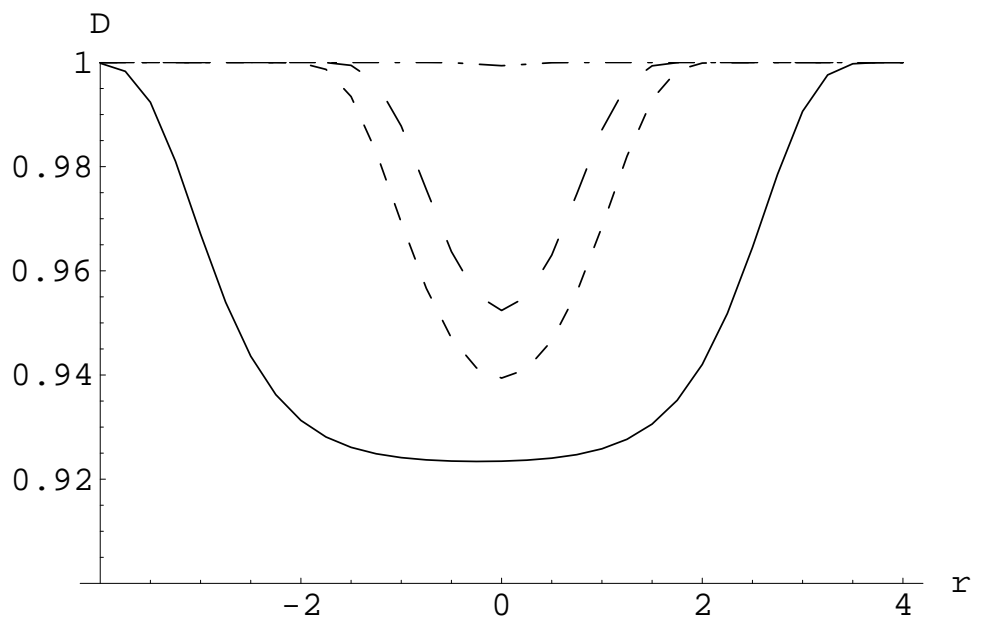

FIG. 10: Dispersion as function of environmental squeezing parameter $r$ for a two-level system starting in an atomic coherent state (Eq. (18)), at various temperatures for a QND system-environment interaction. Here $a=0.0, \gamma_{0}=0.0025, t=1.0$, $\omega=1.0, \omega_{c}=100$, and $\alpha^{\prime}=\beta^{\prime}=\pi / 4$. The bold, small-dashed, large-dashed and dot-dashed curves correspond to temperatures (in units where $\hbar \equiv k_{B}=1$ ) $T=0,50,100$ and 1000, respectively.

the bath exposure time $(t)$ tends to shift and level out the distribution pattern. For finite temperatures, the latter effect predominates, and one observes a steady leveling out with time, with dispersion $D$ tending to 1 . Interestingly, the use of variance in place of dispersion for the data in Figure 11 results in a qualitatively different behavior.

\section{CONCLUSIONS}

In this paper quantum phase distributions of a number of physically interesting systems, interacting with their environment via a QND or a dissipative type of coupling, are analyzed. The system has been taken to be either a two-level atom (or equivalently, a spin-1/2 system) or a harmonic oscillator with the environment being modeled as a bath of harmonic oscillators, initially in a squeezed thermal state, from which the common thermal bath results may be easily extracted by setting the squeezing parameters to zero. The phase distributions are explicitly evaluated taking into account the effect of the different environmental parameters on the dynamics of the system starting from 


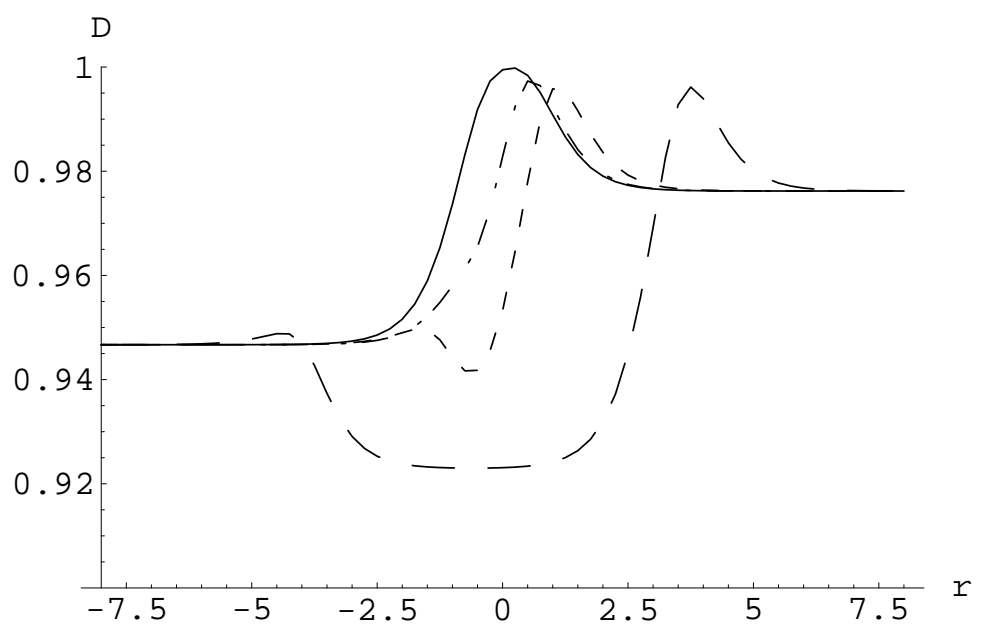

FIG. 11: Dispersion as function of environmental squeezing parameter $r$ for a two-level system starting in an atomic coherent state (Eq. (18) ), at various temperatures for a dissipative system-environment interaction. Here $\gamma_{0}=0.0025, t=1.0, \omega=1.0$, $\omega_{c}=100.0, \Phi=\pi / 8$ [Eq. (44)], and $\alpha^{\prime}=\beta^{\prime}=\pi / 4$. The large-dashed, small-dashed, dot-dashed and bold curves correspond to temperatures $T$ (in units where $\hbar \equiv k_{B}=1$ ) $0,100,300$ and 1000, respectively.

various initial states.

In Section II, we recalled previous work on phase distributions for QND systems [36] of two-level atomic systems (Section IIA) for different initial conditions of the system, starting (1) in an atomic coherent state, and (2) in an atomic squeezed state; and also of a harmonic oscillator (Section IIB) with the oscillator starting initially in (1) a coherent state, and (2) a squeezed coherent state. In Section IIA, some of the above results were extended by considering the phase distribution for multiple two-level atoms. In particular we studied, in Figure1, the effect of the environmental parameters on the distribution for ten atoms starting in an atomic squeezed state and undergoing a QND system-bath interaction. The increase in bath squeezing $r$ and temperature $T$ causes phase diffusion while the increase in the bath exposure time $t$, causes the phase distribution to diffuse as well as shift. The phase distributions are normalized. We also introduced the number distribution $p(m)$, expectation of the reduced density matrix $\rho^{s}(t)$ in the Wigner-Dicke states $|j, m\rangle$. By regarding the variables $m$ and $\phi$ as the 'number' and 'phase' of the atomic system, the relationship between the distributions $p(m)$ and $P(\phi)$ may be considered as expressing complementarity in an atomic context.

In Section III, the reduced density matrix of a two-level system interacting with a squeezed thermal bath via a dissipative system-bath interaction, resulting in a Lindblad form of evolution, was obtained, which reduces to the one found by Nakazato et al. [59] for the case of a thermal bath without squeezing. This solution was used to study the phase distribution for the system, starting (1) in an atomic coherent state, and (2) in an atomic squeezed state. The phase distribution curves preserve the normalization of the distribution. The phase distribution exhibit diffusion as well as shift with time, as seen from Figures 2 and 5 . An interesting feature that emerges from our work is that the relationship between squeezing and temperature effects depend on the type of system-bath interaction. In the case of QND type interaction, squeezing and temperature work in tandem, and produce a diffusive effect on the phase distribution. In contrast, in case of a dissipative interaction, with the reduced system dynamics governed by a Lindblad equation (40), squeezing tends to counteract the influence of temperature, manifesting as a resistence to randomization of phase. This was noted for example in a comparison between the large- and dot-dashed curves of Figure 2, and also in comparison between the bold and large-dashed curves in Figures 5 . A similar behavior is observed in the joint effect of temperature and squeezing on the geometric phase of a qubit (two-level system) interacting dissipatively with its environment [40]. Complementarity between the variables $m$ and $\phi$, by a comparison of the distributions $p(m)$ and $P(\phi)$, was brought out in an interesting manner for the case of a dissipative system-environment interaction and seen from a comparison of Figure 4 with Figure 3. In Figure 3, for the case where temperature $T=0$ and bath squeezing parameter $r=0$, the system tends to the pure state $|j=1 / 2, m=-1 / 2\rangle$, as seen by the large-dashed curve. This corresponds to the action of a quantum deleter [43] by means of an amplitude damping channel [44]. Correspondingly the complementary distribution $P(\phi)$ is seen to level out (the large-dashed curve in Figure 4), indicating complete randomization.

In Section IV, the quantum phase distribution for a harmonic oscillator in a dissipative interaction with a squeezed thermal bath, with the system starting out in a squeezed coherent state, was obtained. An interesting fact that emerged was that under the action of the master equation (60), which is of a Lindblad kind, a harmonic oscillator 
starting in a squeezed coherent state ends in a mixture that can be expressed as a sum over GSCS. A comparison of this distribution with that of the analogous case for a QND system-bath interaction (Figure 6) brings out the differing effects of the two types of system-bath interactions on the phase distribution.

In Section $\nabla$, as an application we studied the dispersion of phase using the phase distributions conditioned on particular initial states of the system. In the case of QND system-bath interaction, the profile of dispersion $D$ is symmetric about $r=0$, as seen from Figures 7 , 9 and 10. In contrast, the profile of dispersion is not symmetric in the case of a dissipative interaction (Figure 11), indicative of the greater complexity of the latter type of interaction. Dispersion is a measure of phase fluctuations. Since the phase distributions used here are obtained taking the effect of the environment into consideration, the dispersions calculated using them would set a realistic estimate on phase measurements in a number of experimental scenarios.

We hope that the treatment of phase distributions developed here would be of interest both from a technical point of view as well as in the context of experimental situations.

\section{Acknowledgments}

We thank Prof. Joachim Kupsch for helpful comments. We are also thankful to the anonymous Referee for suggestions that have helped improve Section $\nabla$

[1] W. H. Louisell, Quantum Statistical Properties of Radiation (John Wiley and Sons, 1973).

[2] A. O. Caldeira and A. J. Leggett, Physica A 121, 587 (1983).

[3] W. H. Zurek, Phys. Today 44, 36 (1991); Prog. Theor. Phys. 87, 281 (1993).

[4] J. Shao, M-L. Ge and H. Cheng, Phys. Rev. E 53, 1243 (1996).

[5] D. Mozyrsky and V. Privman, Journal of Stat. Phys. 91, 787 (1998).

[6] G. Gangopadhyay, M. S. Kumar and S. Dattagupta, J. Phys. A: Math. Gen. 34, 5485 (2001).

[7] H.-P. Breuer and F. Petruccione, The Theory of Open Quantum Systems (Oxford University Press, 2002).

[8] V. Hakim and V. Ambegaokar, Phys. Rev. A 32, 423 (1985).

[9] C. M. Smith and A. O. Caldeira, Phys. Rev. A 36, 3509 (1987); ibid 41, 3103 (1990).

[10] H. Grabert, P. Schramm and G. L. Ingold, Phys. Rep. 168, 115 (1988).

[11] S. Banerjee and R. Ghosh, Phys. Rev. A 62, 042105 (2000).

[12] S. Banerjee and R. Ghosh, Phys. Rev. E 67, 056120 (2003).

[13] C. J. Myatt, B. E. King, Q. A. Turchette, C. A. Sackett, et al., Nature 403, 269 (2000).

[14] Q. A. Turchette, C. J. Myatt, B. E. King, C. A. Sackett, et al., Phys. Rev. A 62, 053807 (2000).

[15] G. J. Pryde, J. L. O’Brien, A. G. White, et al., Phys. Rev. Lett. 92, 190402 (2004); J. L. O'Brien, G. J. Pryde, A. G. White, et. al., Nature 426, 264 (2003).

[16] R. Onofrio and L. Viola, Phys. Rev. A 58, 69 (1998).

[17] A. Kuzmich, N. P. Bigelow and L. Mandel, Europhys. Lett. 42, 481 (1998).

[18] J. Clausen, J. Salo, V. M. Akulin and S. Stenholm, Phys. Rev. A 72, 062104 (2005).

[19] S. Banerjee and R. Ghosh, J. Phys. A: Math. Theo. 40, 1273 (2007).

[20] V. B. Braginsky, Yu. I. Vorontsov and K. S. Thorne, Science 209, 547 (1980).

[21] V. B. Braginsky and F. Ya. Khalili, in Quantum Measurements, edited by K. S. Thorne (Cambridge University Press, Cambridge, 1992).

[22] D. F. Walls and G. J. Milburn, Quantum Optics (Springer, Berlin, 1994).

[23] W. H. Zurek, in The Wave-Particle Dualism, edited by S. Diner, D. Fargue, G. Lochak and F. Selleri (D. Reidel Publishing Company, Dordrecht, 1984).

[24] C .M. Caves, K. D. Thorne, R. W. P. Drever, V. D. Sandberg and M. Zimmerman, Rev. Mod. Phys. 52, 341 (1980).

[25] M. F. Bocko and R. Onofrio, Rev. Mod. Phys. 68, 755 (1996).

[26] W. P. Schleich and S. M. Barnett (eds.), Quantum Phase and Phase Dependent Measurements, Physica Scripta Special issue T48 (1993).

[27] V. Peřinová, A. Lukš and J. Peřina, Phase in Optics (World Scientific, Singapore, 1998).

[28] P. A. M. Dirac, Proc. R. Soc. Lond. A 114, 243 (1927).

[29] L. Susskind and J. Glogower, Physics 1, 49 (1964).

[30] P. Carruthers and M. M. Nieto, Rev. Mod. Phys. 40, 411 (1968).

[31] D. T. Pegg and S. M. Barnett, J. Mod. Opt. 36, 7 (1989); Phys. Rev. A 39, 1665 (1989).

[32] J. H. Shapiro, S. R. Shepard and N. C. Wong, Phys. Rev. Lett. 62, 2377 (1989).

[33] J. H. Shapiro and S. R. Shepard, Phys. Rev. A 43, 3795 (1991).

[34] M. J. W. Hall, Quantum Opt. 3, 7 (1991).

[35] G. S. Agarwal, S. Chaturvedi, K. Tara and V. Srinivasan, Phys. Rev. A 45, 4904 (1992).

[36] S. Banerjee, J. Ghosh and R. Ghosh, Phys. Rev. A 75, 062106 (2007); eprint quant-ph/0703055 
[37] T. A. B. Kennedy and D. F. Walls, Phys. Rev. A 37, 152 (1988).

[38] M. S. Kim and V. Bužek, Phys. Rev. A 47, 610 (1993).

[39] S. Banerjee and R. Ghosh, J. Phys. A: Math. and Theo. 40, 13735 (2007); eprint quant-ph/0703054.

[40] S. Banerjee and R. Srikanth, to appear in Eur. Phys. J. D; eprint quant-ph/0611161.

[41] G. S. Agarwal and R. P. Singh, Phys. Lett. A 217, 215 (1996).

[42] I. Gradstein and I. Ryzhik, Table of Integrals, Series, and Products (Academic Press, New York, 1980).

[43] R. Srikanth and S. Banerjee, Phys. Lett. A 367, 295 (2007); eprint quant-ph/0611263

[44] M. Nielsen and I. Chuang, Quantum Computation and Quantum Information (Cambridge University Press, Cambridge, 2000).

[45] W. G. Unruh, Phys. Rev. A 51, 992 (1995).

[46] G. M. Palma, K-A. Suominen and A. K. Ekert, Proc. R. Soc. Lond. A 452, 567 (1996).

[47] D. P. DiVincenzo, Phys. Rev. A 51, 1015 (1995).

[48] R. H. Dicke, Phys. Rev. 93, 99 (1954).

[49] F. T. Arecchi, E. Courtens, R. Gilmore and H. Thomas, Phys. Rev. A 6, 2211 (1972).

[50] J. M. Radcliffe, J. Phys. A: Gen. Phys. 4, 313 (1971).

[51] M. A. Rashid, J. Math. Phys. 19, 1391 (1978).

[52] G. S. Agarwal and R. R. Puri, Phys. Rev. A 41, 3782 (1990).

[53] J. P. Dowling, G. S. Agarwal and W. P. Schleich, Phys. Rev. A 49, 4101 (1994).

[54] C. Aragone, E. Chalbaud and S. Salamo, J. Math. Phys. 17, 1963 (1976).

[55] D. A. Varshalovich, A. N. Moskalev and V. K. Khersonskii, Quantum Theory of Angular Momentum (World Scientific, Singapore, 1988).

[56] R. Srikanth and S. Banerjee, in preparation.

[57] M. O. Scully and M. S. Zubairy, Quantum Optics (Cambridge University Press, Cambridge, 1997).

[58] R. Srikanth and S. Banerjee, eprint arXiv:0707.0059

[59] H. Nakazato, Y. Hida, K. Yuasa, B. Militello, et al., Phys. Rev. A 74, 062113 (2006).

[60] H.-X. Lu, J. Yang, Y.-D. Zhang and Z-B. Chen, Phys. Rev. A 67, 024101 (2003).

[61] A. K. Ekert and P. L. Knight, Phys. Rev. A 42, 487 (1990).

[62] S. M. Roy and V. Singh, Phys. Rev. D 25, 3413 (1982).

[63] M. V. Satyanarayana, Phys. Rev. D 32, 400 (1985); P. Marian, Phys. Rev. A 44, 3325 (1991).

[64] S. Banerjee and J. Kupsch, J. Phys. A: Math. Gen. 38, 5237 (2005).

[65] J. Kupsch and S. Banerjee, Inf. Dim. Anal., Quantum Probability and Related Topics 9, 413 (2006).

[66] A. Erdelyi, W. Magnus, F. Oberhettinger and F. G. Tricomi, Higher Transcendental Functions, Vol. I (McGraw-Hill, New York, 1953).

[67] A. Bandilla and H. Paul, Ann. Phys. (Leipzig) 23, 323 (1969); A. Bandilla, H.Paul and H.-H. Ritze, Quantum Opt. 3, 267 (1991).

[68] T. Opartný, J. Phys. A: Math. Gen. 27, 7201 (1994).

[69] A. Lukš and V. Peřinová, J. Phys. A: Math. Gen. 29, 4665 (1996). 